\title{
Spectral Analyses and Radiation Exposures from Several Ground-Level Enhancement (GLE) Solar Proton Events: A Comparison of Methodologies
}

\author{
William Atwell ${ }^{1}$ \\ The Boeing Company, Houston, TX, 77059 USA \\ Allan Tylka ${ }^{2}$ \\ Naval Research Laboratory, Washington, DC 20375 USA \\ William Dietrich ${ }^{3}$ \\ Consultant, Prospect Hts., IL 60070 USA \\ Francis Badavi ${ }^{4}$ \\ Christopher Newport University, Hampton, VA 23681 USA \\ Kristina Rojdev ${ }^{5}$ \\ NASA Johnson Space Center, Houston, TX 77058 USA
}

\begin{abstract}
Several methods for analyzing the particle spectra from extremely large solar proton events, called Ground-Level Enhancements (GLEs), have been developed and utilized by the scientific community to describe the solar proton energy spectra and have been further applied to ascertain the radiation exposures to humans and radio-sensitive systems, namely electronics. In this paper 12 GLEs dating back to 1956 are discussed, and the three methods for describing the solar proton energy spectra are reviewed. The three spectral fitting methodologies are EXP [an exponential in proton rigidity (R)], WEIB [Weibull fit: an exponential in proton energy], and the Band function (BAND) [a double power law in proton rigidity]. The EXP and WEIB methods use low energy (MeV) GLE solar proton data and make extrapolations out to $\sim 1 \mathrm{GeV}$. On the other hand, the BAND method utilizes low- and medium-energy satellite solar proton data combined with high-energy solar proton data deduced from high-latitude neutron monitoring stations. Thus, the BAND method completely describes the entire proton energy spectrum based on actual solar proton observations out to $\sim 10 \mathrm{GeV}$. Using the differential spectra produced from each of the 12 selected GLEs for each of the three methods, radiation exposures are presented and discussed in detail. These radiation exposures are then compared with the current 30-day and annual crew exposure limits and the radiation effects to electronics.
\end{abstract}

\section{Nomenclature}

$\begin{array}{ll}c S V & =\text { centi Sievert } \\ G L E & =\text { Ground Level Enhancement } \\ G O E S & =\text { Geostationary Operational Environmental Satellite } \\ H E P A D & =\text { High Energy Proton and Alpha Detector } \\ M E P A D & =\text { Medium Energy Proton and Alpha Detector } \\ S P E & =\text { Solar Proton Event }\end{array}$

\footnotetext{
${ }^{1}$ Technical Fellow, Boeing Research \& Technology, 13100 Space Center Blvd./HB 2-30, AIAA Associate Fellow.

${ }^{2}$ Scientist, E. O. Hulburt Center for Space Research, Naval Research Laboratory, Washington, DC 20375.

${ }^{3}$ Consultant, 13 S. Parkway, Prospect Heights., IL 60070.

${ }^{4}$ Associate Professor, Christopher Newport University, Hampton, VA 23681.

${ }^{5} \mathrm{Ph}$ D candidate, USC, Los Angeles, CA, and Aerospace Engineer, NASA Johnson Space Center, Houston, TX 77058, AIAA Student Member.
} 


\section{Introduction}

Solar protons events (SPEs) represent the single-most significant source of acute radiation exposure to humans

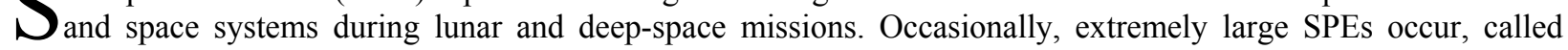
Ground Level Enhancements (GLEs), that contain protons having energies extending into the GeV range. Early in the "Space Age era," particle detectors on satellites had limited energy resolution capability. Malitson and Webber ${ }^{1}$ suggested an exponential in proton rigidity fit as a means of describing the energy spectrum of an SPE based on two data points: integral fluence $\Phi>30 \mathrm{MeV}$ and $>100 \mathrm{MeV}$ (protons $/ \mathrm{cm}^{2}$ ). Beyond $100 \mathrm{MeV}$ the particle energy spectrum was extrapolated out to several $\mathrm{GeV}$. This methodology was used by the scientific community for several decades. Later, an energy spectrum fitting method, called a Weibull fit, was introduced by Xapsos, et al. ${ }^{2}$. Most recently, Tylka and Dietrich ${ }^{3}$ and Atwell, et al. ${ }^{4}$ applied a Band function ${ }^{5}$ fit, which is a double power law in proton rigidity. Their method is based on actual proton data observed from medium (10-100 MeV) and high (several hundred $\mathrm{MeV}$ ) proton energies (GOES MEPAD and HEPAD and other satellites) and from cascade secondary neutrons produced during nuclear collisions of the incoming solar protons with the Earth's atmosphere ( $>1 \mathrm{GV})$. These neutrons are measured at the high latitudes by a network of neutron monitoring stations. These authors have successfully applied the Band function fitting method to 70 GLEs dating back to 1956. Since this method completely describes the entire proton energy spectrum for an event, this is the method that is strongly suggested that the scientific community use for future GLE SPE assessment and analysis.

In this paper we have selected 12 GLEs from the original $\sim 70$ GLEs and present the integral and differential proton energy spectra ranging from $10 \mathrm{MeV}$ to $5 \mathrm{GeV}$. In addition, we have taken the differential spectra and computed "skin" and "Blood-Forming Organ (BFO)" radiation exposures using the NASA Langley Research Center HZETRN $2010^{6}$ high energy particle transport/dose code. These skin and BFO exposures for the 12 GLEs are compared with the current NASA 30-day and annual crew exposure limits.

\section{The Three Fitting Methods}

\section{A. The Exponential in Proton Rigidity Method (EXP)}

The exponential in particle rigidity fitting method of Malitson and Webber ${ }^{1}$ is based on two proton integral data points: $>30 \mathrm{MeV}$ and $>100 \mathrm{MeV}$. The mathematical expression for the integral energy spectrum is:

$$
\Phi(>\mathrm{E})=\mathrm{N}_{\mathrm{o}} * \exp \left(-\mathrm{R} / \mathrm{R}_{\mathrm{o}}\right)
$$

where $\Phi(>E)$ is the integral energy fluence in protons $/ \mathrm{cm}^{2}, \mathrm{~N}_{\mathrm{o}}$ is a normalization constant, $\mathrm{R}$ is the proton rigidity (proton momentum) in MV (million volts), and $R_{0}$ is the characteristic rigidity in MV. The proton rigidity $R$ is related to the proton energy $(\mathrm{MeV})$ by:

$$
R(M V)=\sqrt{ }\left(E^{2}-2 * m_{0} * E\right)
$$

where $\mathrm{E}$ is the proton energy $(\mathrm{MeV})$ and $\mathrm{m}_{\mathrm{o}}$ is the rest mass of the proton $(938 \mathrm{MeV})$.

\section{B. The Weibull Function Fitting Method (WEIB)}

Xapsos, et al. ${ }^{2}$ applied a Weibull function to several SPEs that has the mathematical integral form:

$$
\Phi=\Phi_{\mathrm{o}} * \exp \left(-\mathrm{k}^{*} \mathrm{E}^{\alpha}\right)
$$

where $\Phi$ can be either the proton fluence or proton flux having energy that exceeds a threshold energy $\mathrm{E}(\mathrm{MeV})$. The constants $\Phi_{0}, \mathrm{k}$, and $\alpha$ are determined by a nonlinear regression fit to the energy spectrum of interest. The differential energy spectrum is easily found by differentiating with respect to $E$.

$$
\partial \Phi / \partial \mathrm{E}=\Phi_{\mathrm{o}} * \mathrm{k}^{*} \alpha^{*} \mathrm{E}^{\alpha-1} * \exp \left(-\mathrm{k}^{*} \mathrm{E}^{\alpha}\right)
$$

They found that the 3 fitting parameters can easily be extracted from either of the above two equations using a standard Marquardt-Levenberg nonlinear regression routine ${ }^{7,8}$. For several SPEs analyzed by these authors, they show the following Weibull parameters: 
Table 1. Weibull Fitting Parameters for Several SPEs ${ }^{2}$

\begin{tabular}{lllll}
\hline Onset Date & \multicolumn{1}{c}{$\Phi_{Q}$} & \multicolumn{1}{c}{$k$} & $\alpha$ & $r$ \\
\hline & & & & \\
4 August 1972 & $2.455 \times 10^{10}$ & 0.0236 & 1.108 & 0.996 \\
12 August 1989 & $1.622 \times 10^{11}$ & 1.166 & 0.4015 & 0.994 \\
29 Sept. 1989 & $3.631 \times 10^{10}$ & 0.877 & 0.3841 & 0.998 \\
19 October 1989 & $1.230 \times 10^{12}$ & 2.115 & 0.2815 & 0.998 \\
23 March 1991 & $1.660 \times 10^{11}$ & 0.972 & 0.4410 & 0.993 \\
\hline
\end{tabular}

In two other papers, Kim, et al., ${ }^{9,10}$ analyzed a number of SPEs some of which were GLEs using a Weibull distribution of the form

$$
\Phi(>\mathrm{E})=\mathrm{P}_{\mathrm{o}} * \exp \left(-\mathrm{a}^{*} \mathrm{E}^{\mathrm{b}}\right)
$$

We compared the results of two of the events in Table 1 above (29 Sept. 1989 and 19 Oct. 1989) with those given in ref. 9, Table 4, which is shown below:

Table 2. Weibull Fitting Parameters for Several SPEs
\begin{tabular}{|ccccc|}
\hline SPE & $\mathbf{\Phi}(>\mathbf{3 0} \mathbf{M e V})$ & $\mathbf{P}_{\mathbf{o}}$ & $\mathbf{a}$ & \multicolumn{1}{c|}{$\mathbf{b}$} \\
$9 / 29 / 1989$ & $1.35 * 10^{9}$ & $1.90 * 10^{10}$ & 0.5493 & 0.4643 \\
$10 / 19 / 1989$ & $4.23 * 10^{9}$ & $1.91 * 10^{12}$ & 2.5677 & 0.256 \\
\hline
\end{tabular}

It is also noted that Kim, et al. ${ }^{9}$ fitted the SPEs out to a maximum energy value of $1000 \mathrm{MeV}(1 \mathrm{GeV})$.

\section{The Band Function Fitting Method (BAND)}

The fitting method we suggest is that of Tylka and Dietrich ${ }^{3}$ and Atwell, et al. ${ }^{4}$ where $\sim 70$ GLEs dating back to 1956 were fitted using a Band function ${ }^{5}$, (thoroughly discussed in the above two references) which is a double power law in proton rigidity. This method fits actual solar proton data over the entire SPE energy spectrum ranging from $10 \mathrm{MeV}$ to $5 \mathrm{GeV}$.

\section{12 Ground Level Enhancements (GLEs)}

The 12 GLEs utilized for analysis in this paper are:

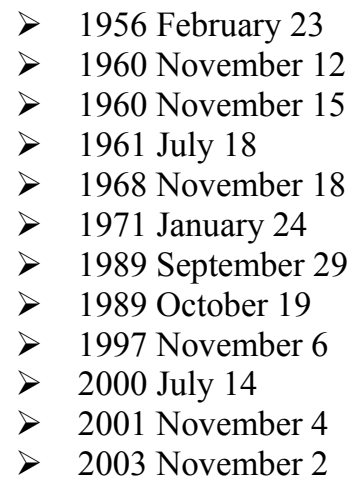

These 12 events were selected, since they were analyzed using a Weibull function by Kim, et al. ${ }^{9}$ and provided a means of comparison with the Band function. The following plots show a comparison of the three fitting methods, Band, exponential and Weibull, for both the integral and differential fitted spectra for the 12 GLEs. Note that we also show a comparison of the combined 1960 November 12 and 1960 November 15 GLEs.

In every case we find that the exponential in proton rigidity fitting method grossly under-estimates both the Band and Weibull fitting methods. 
Comparing the Band method with the Weibull method, we find considerable variation using the Weibull fitting method, which we find rather puzzling. The only reasonable agreement was for the 1956 February 23 GLE, and this may just be a fortuitous fluke. Figures 1. (a) - (m) compare the integral and differential spectra for the GLEs.

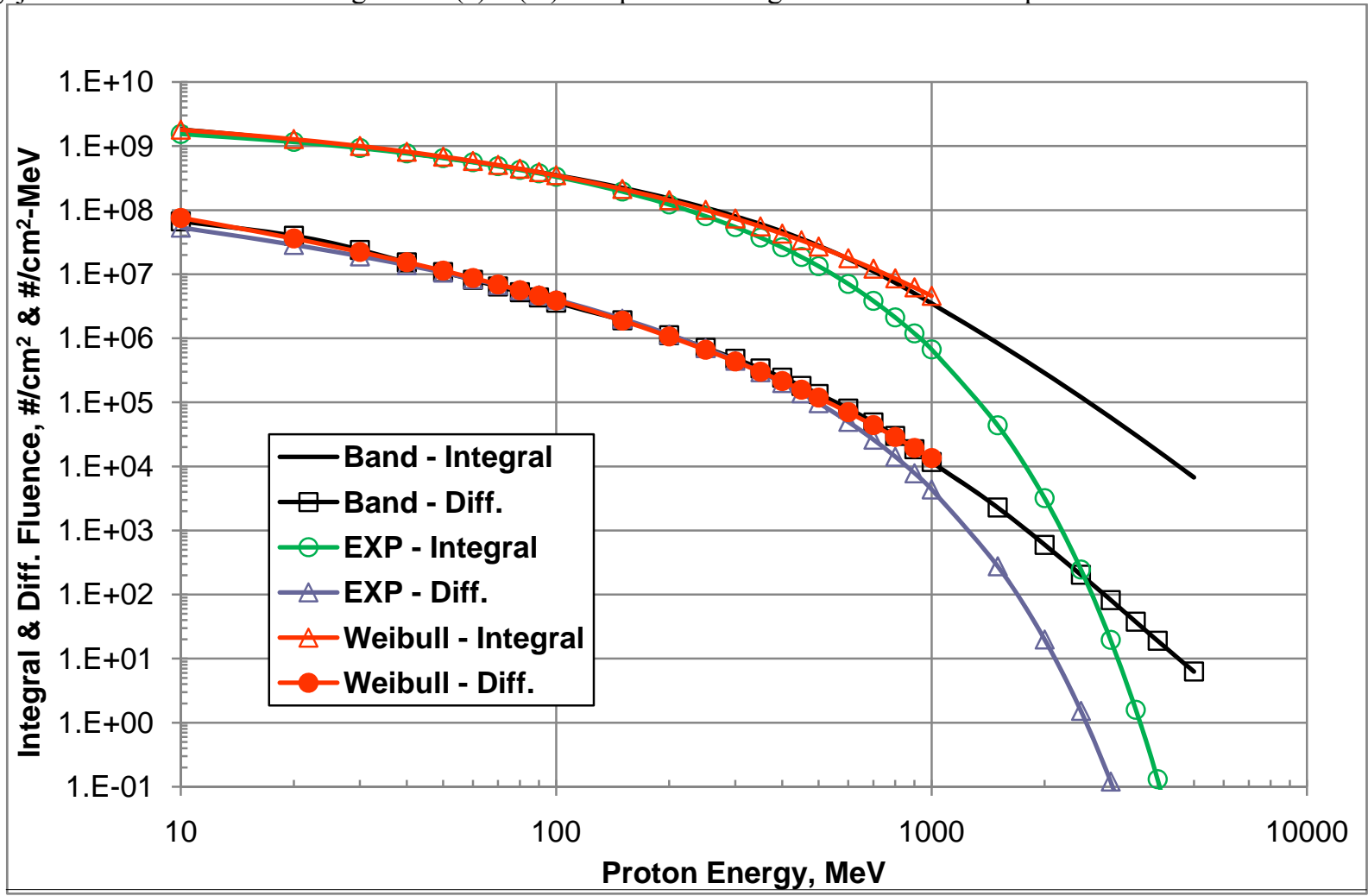

Figure 1 (a) 1956 Feb 23 GLE

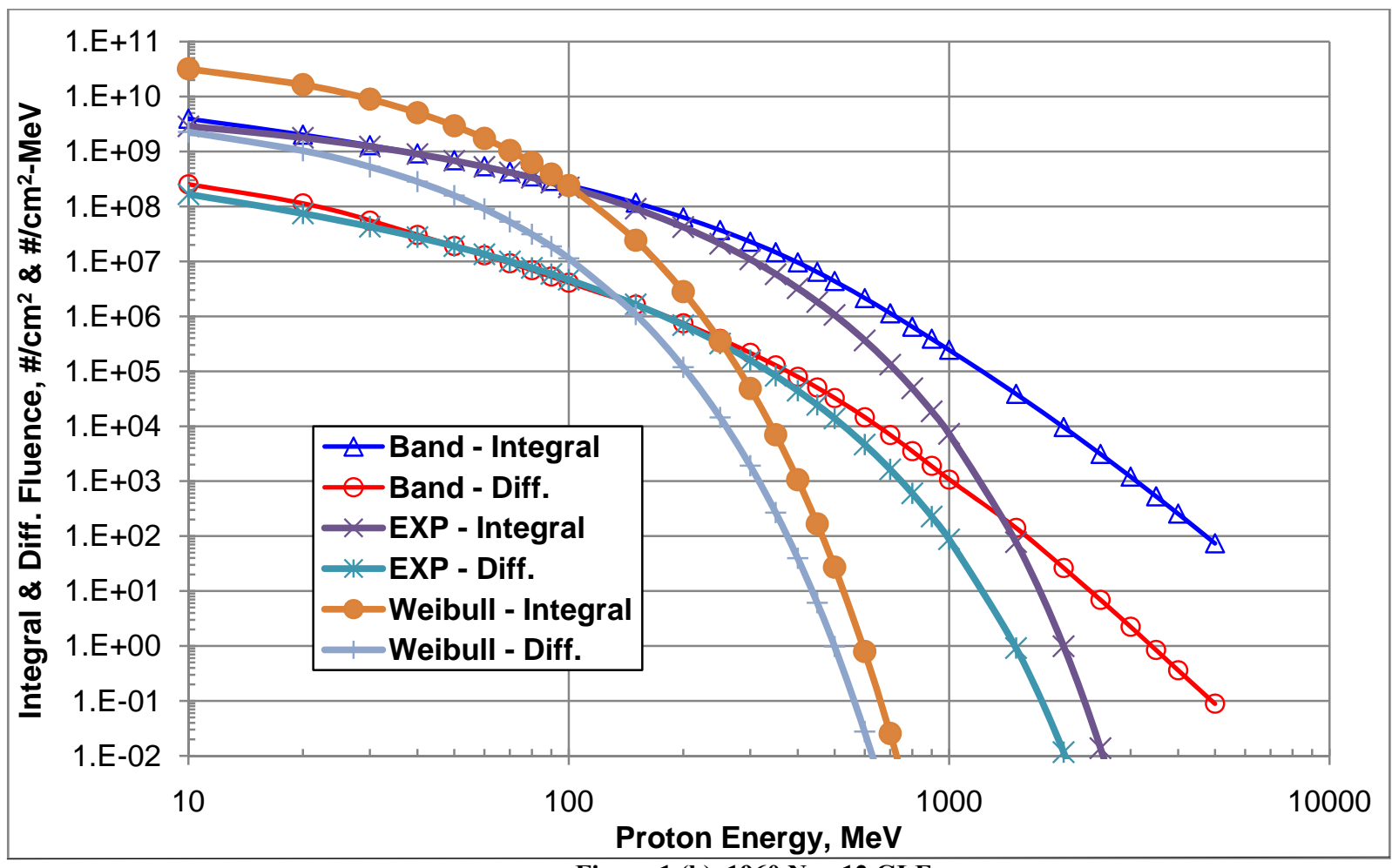

Figure 1 (b) 1960 Nov 12 GLE 


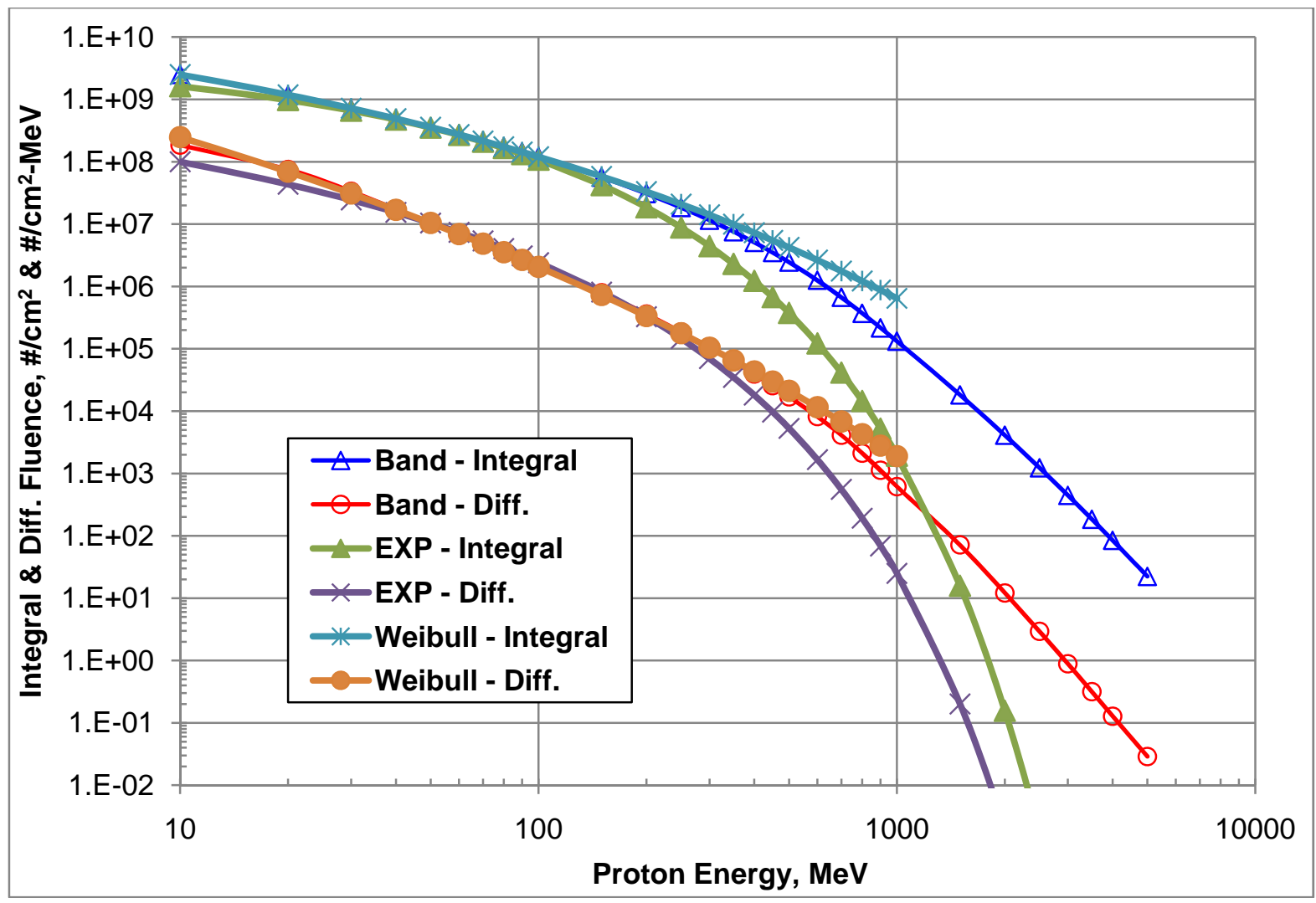

Figure 1 (c) 1960 Nov 15 GLE

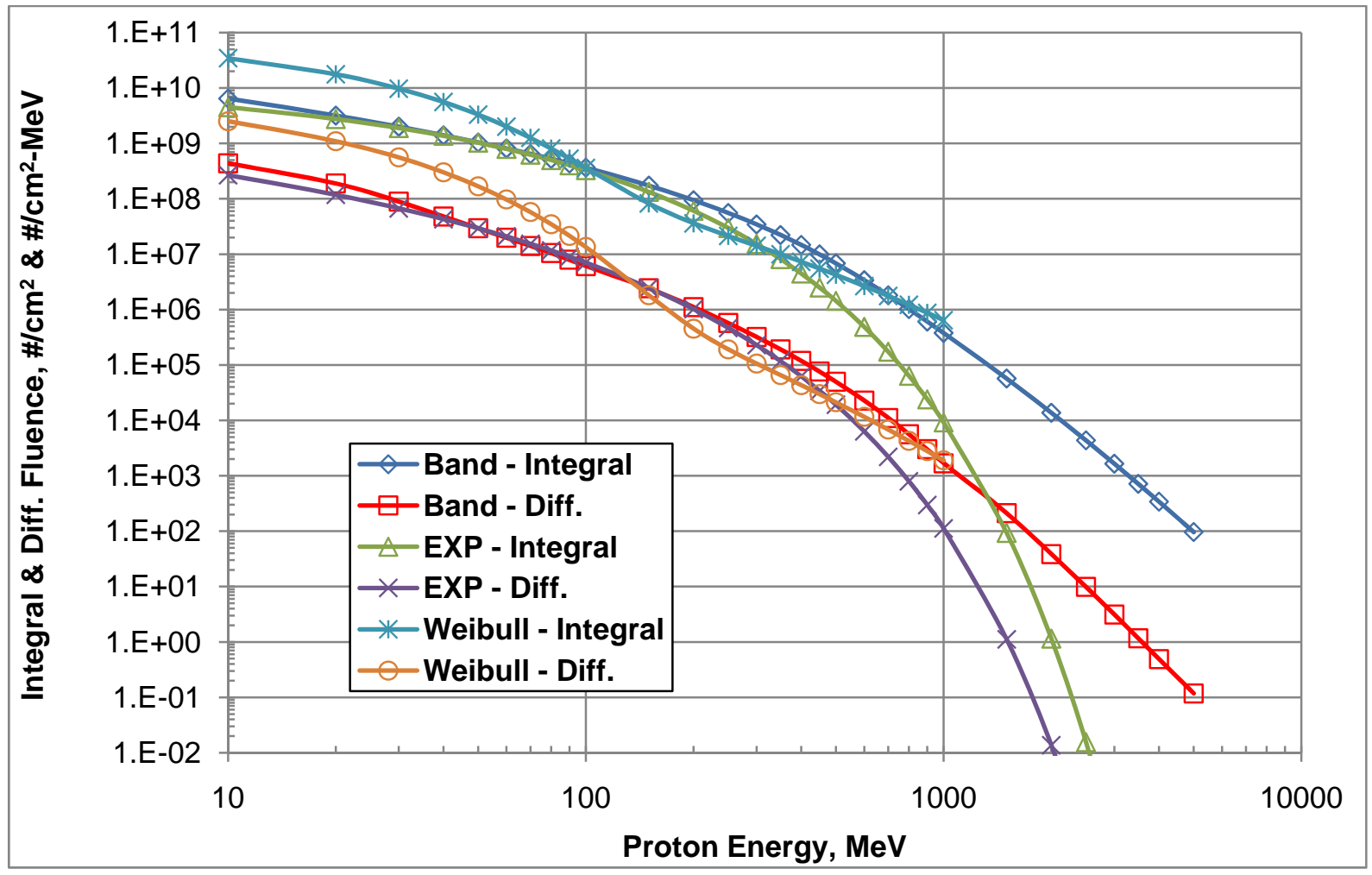

Figure 1 (d) 1960 Nov 12-15 


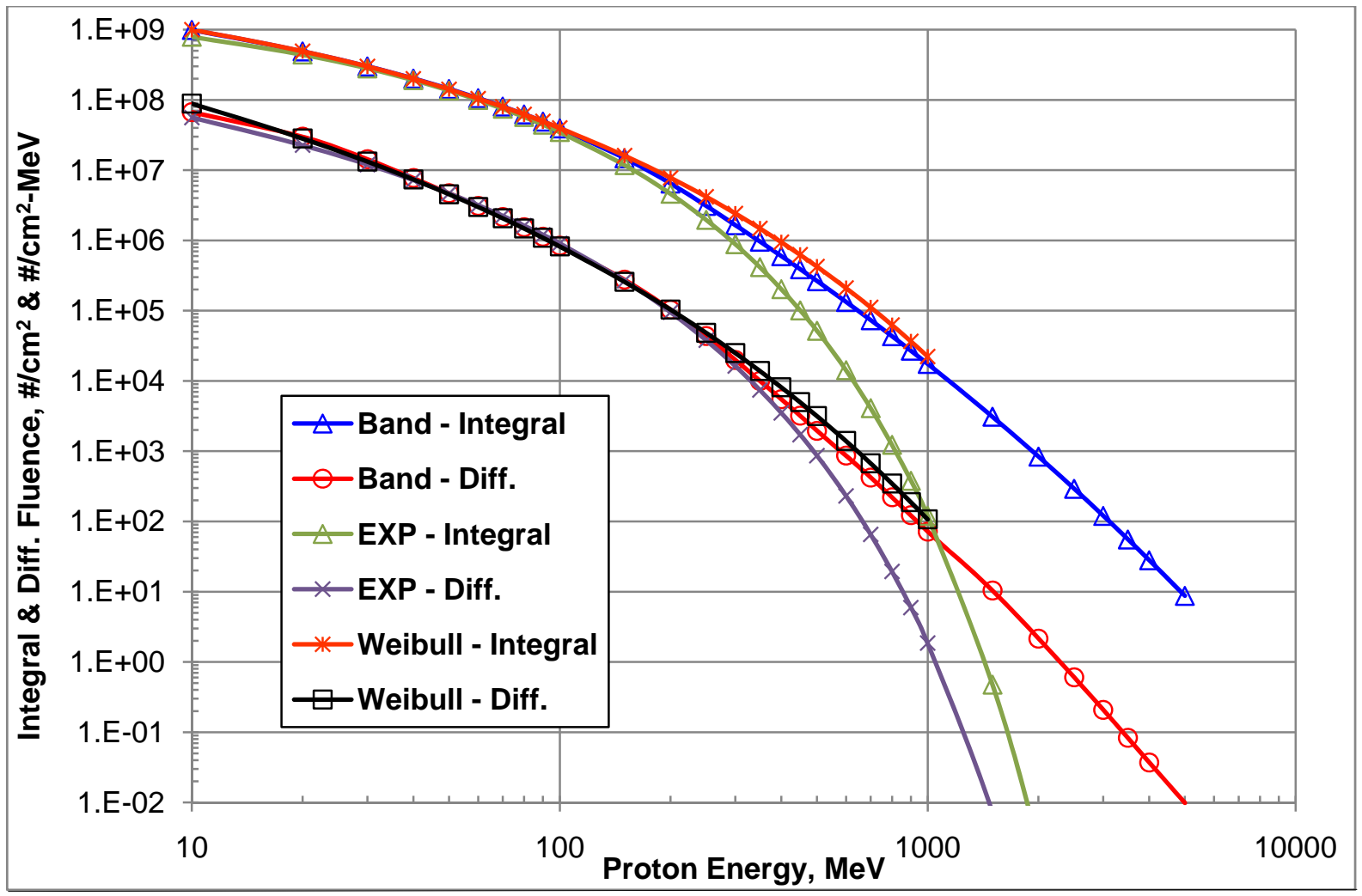

Figure 1 (e) 1961 July 18

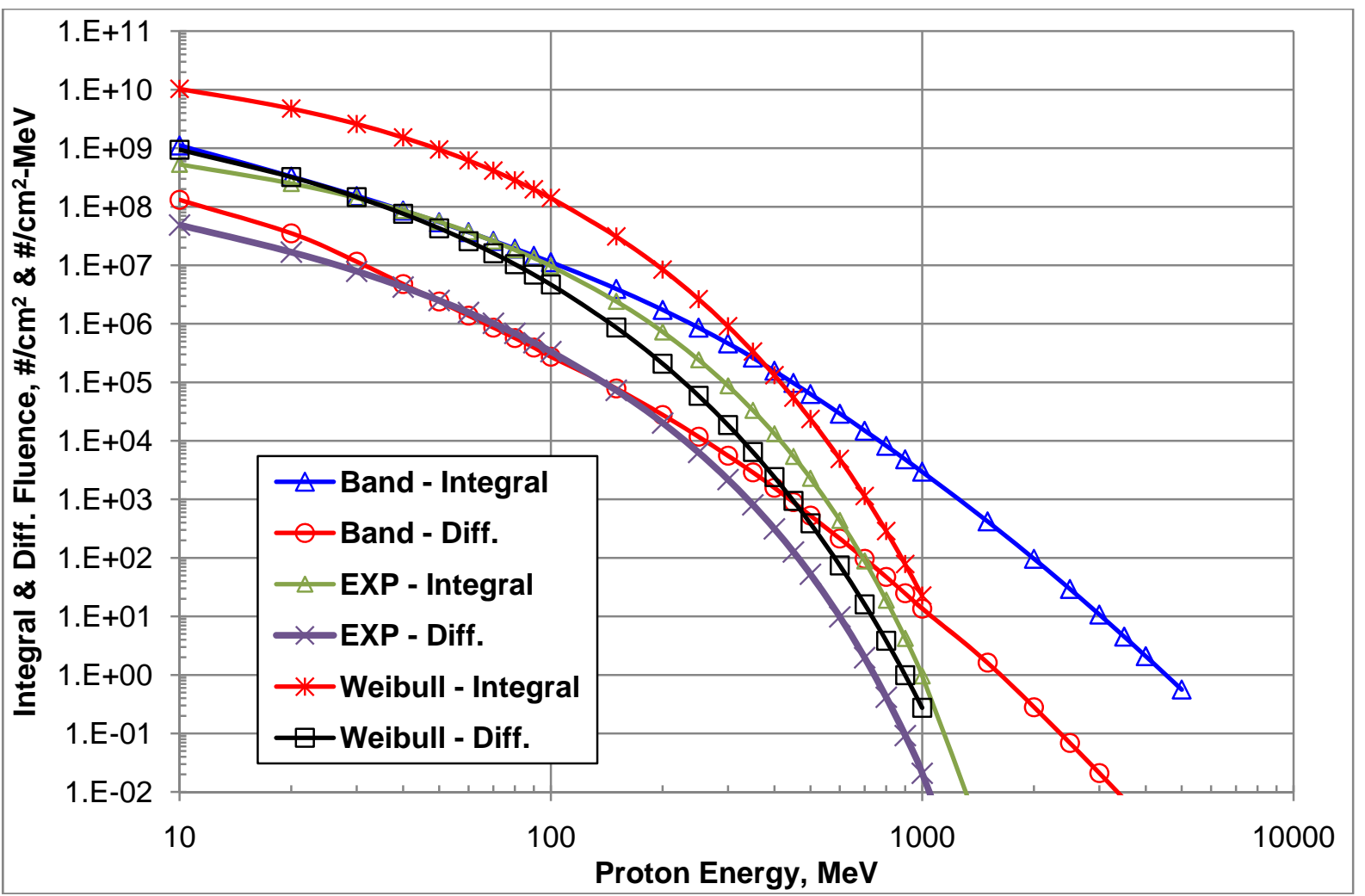

Figure 1 (f) 1968 Nov 18 


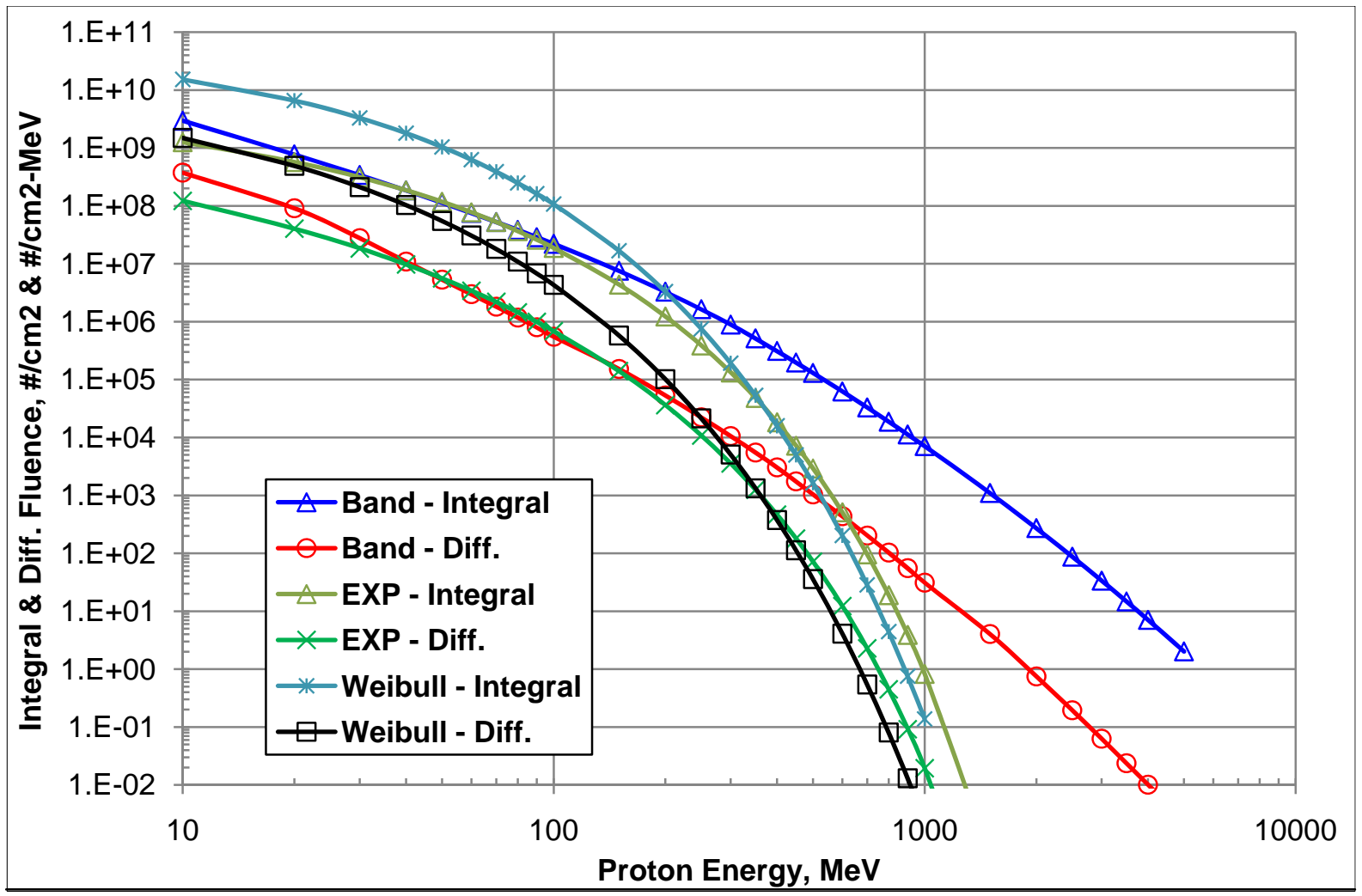

Figure 1 (g) 1971 Jan 24

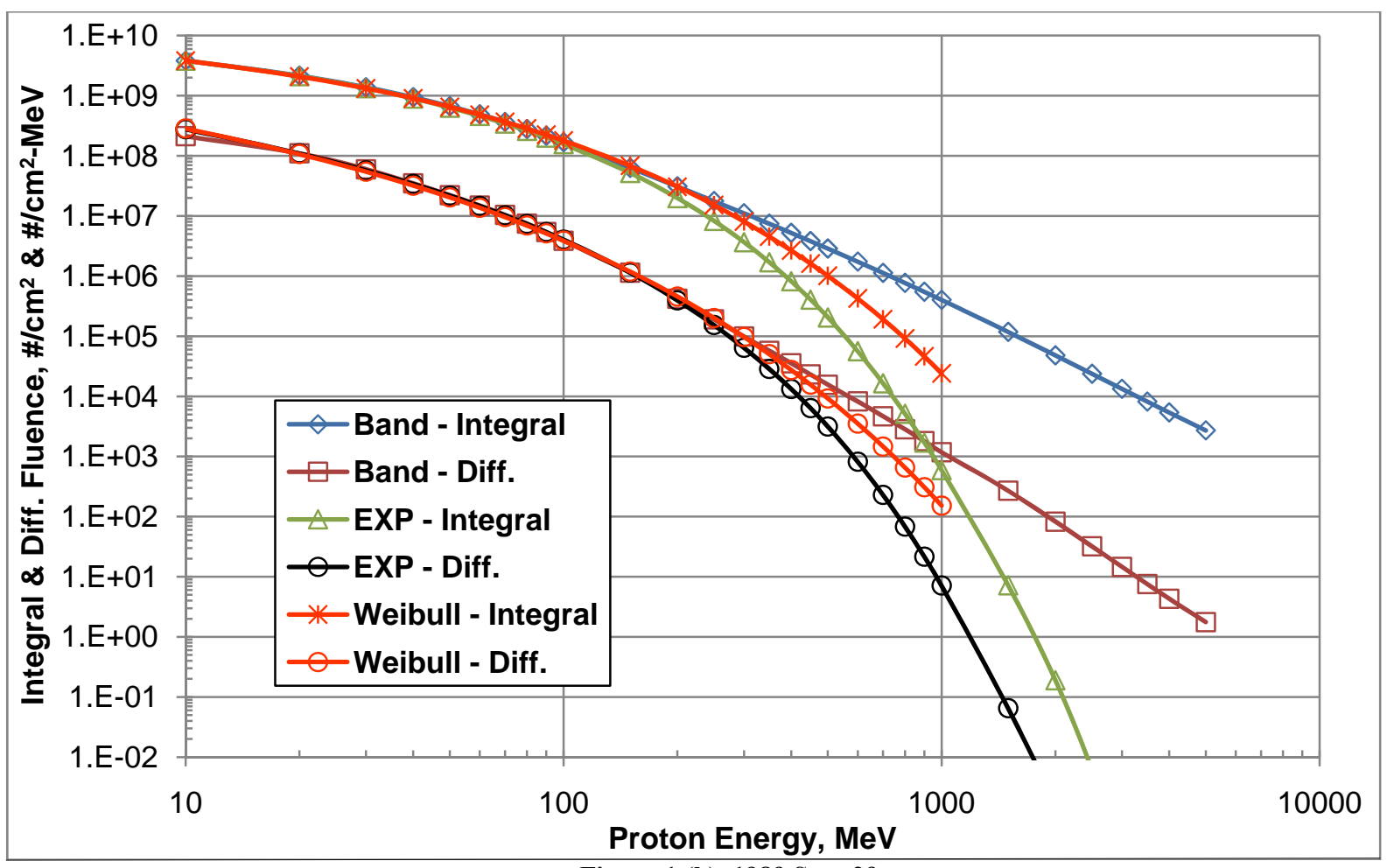

Figure 1 (h) 1989 Sept 29 


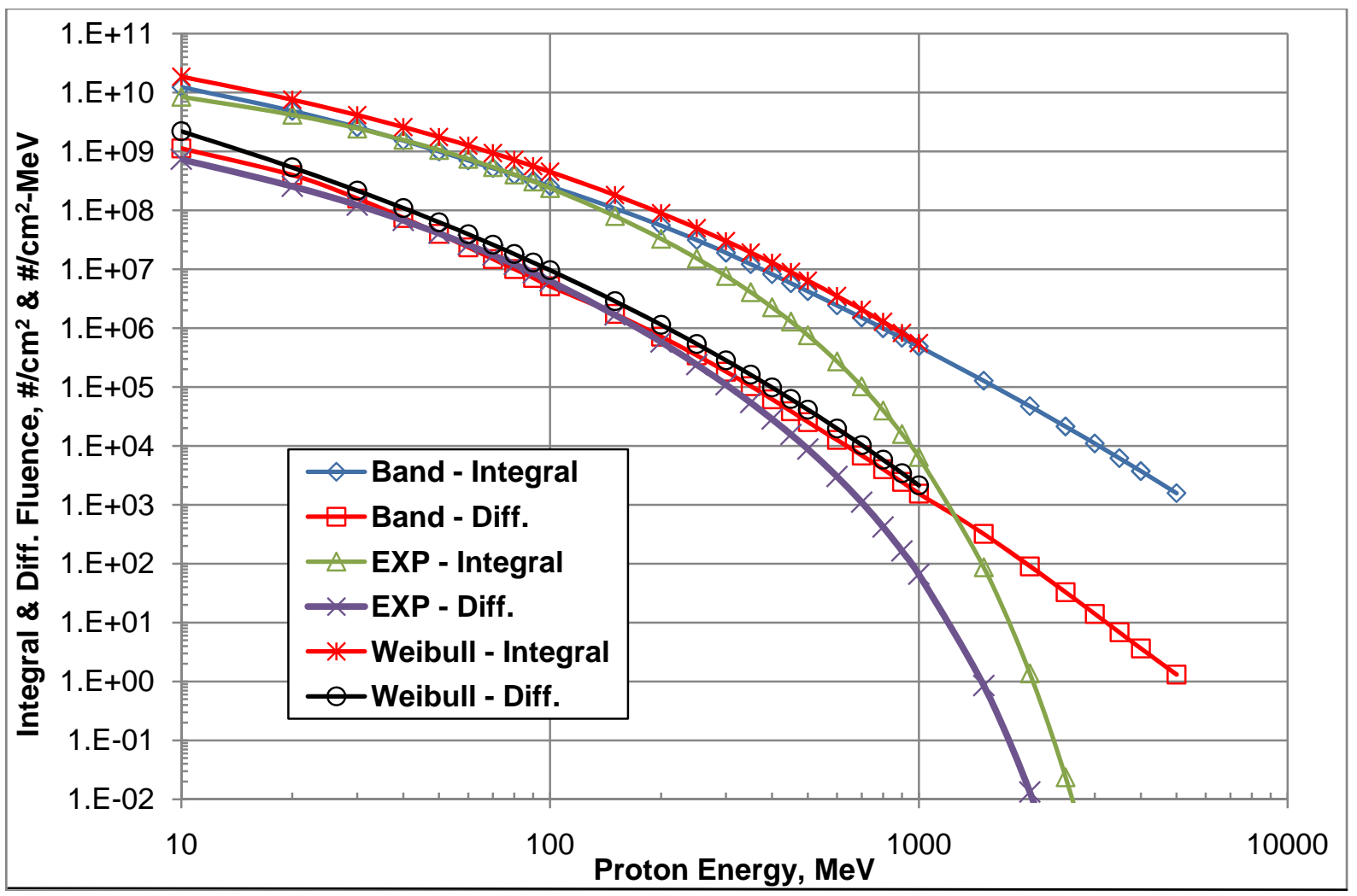

Figure 1 (i) 1989 Oct 19

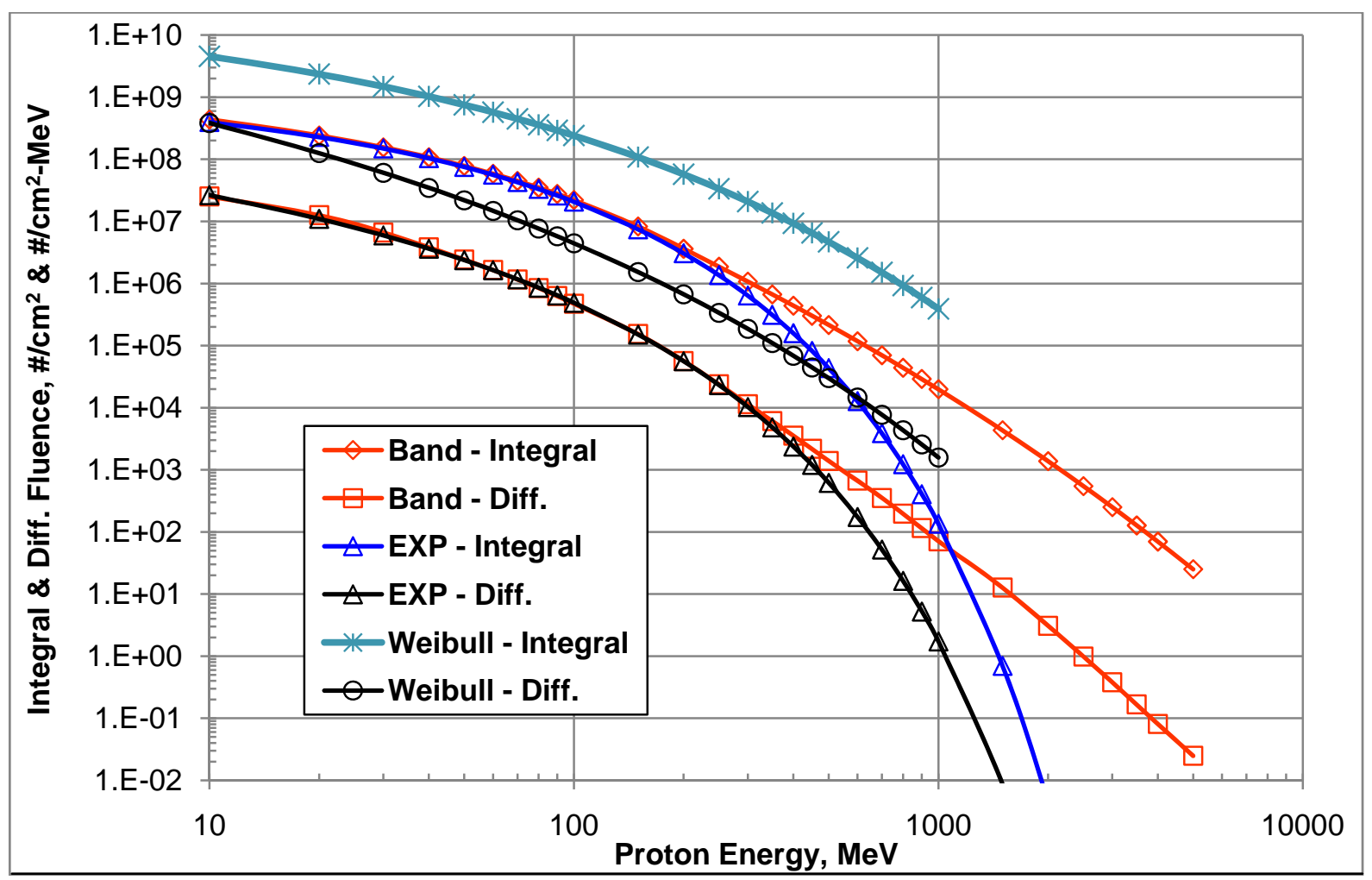

Figure 1 (j) 1997 Nov 6 


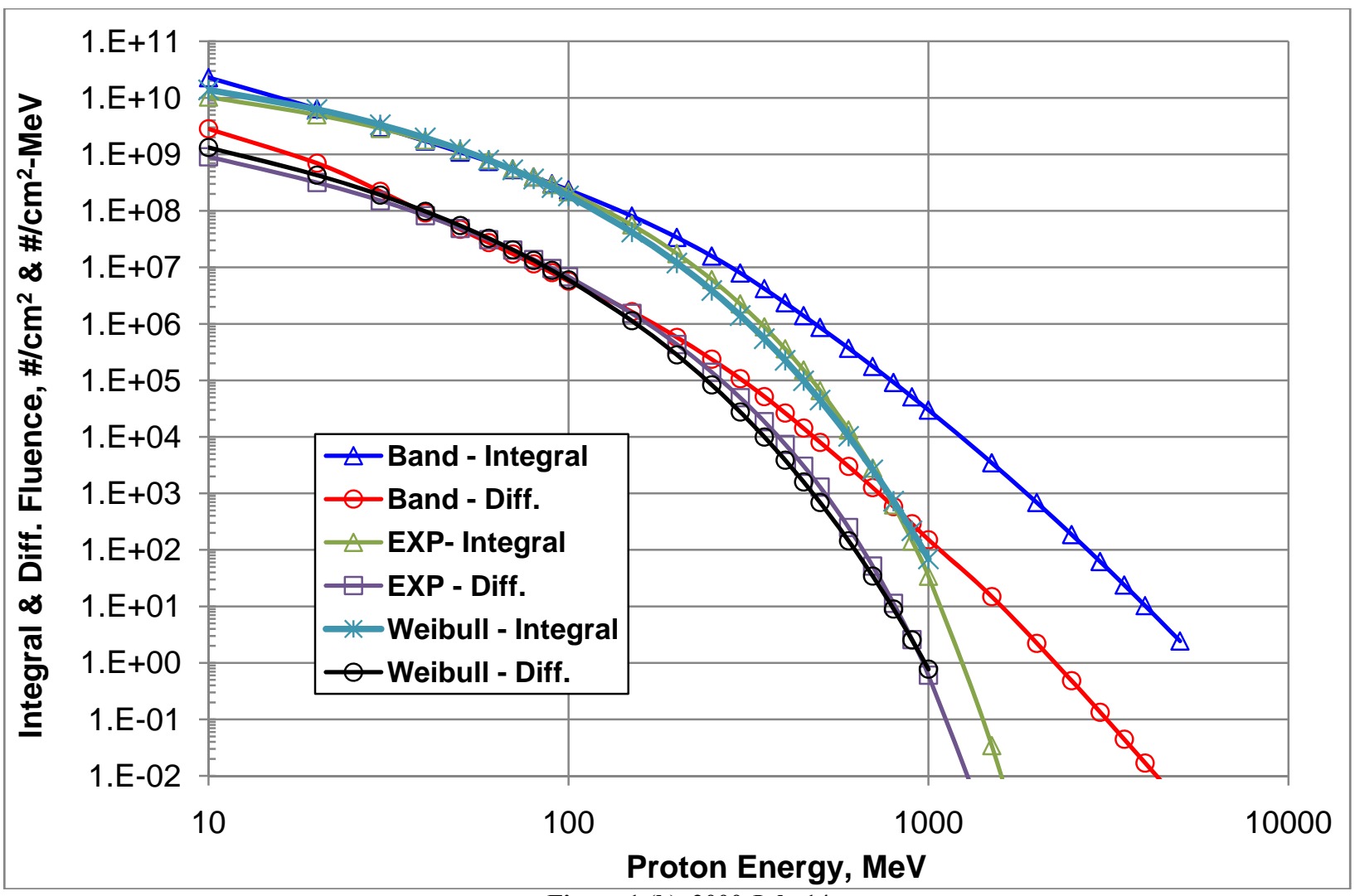

Figure 1 (k) 2000 July 14

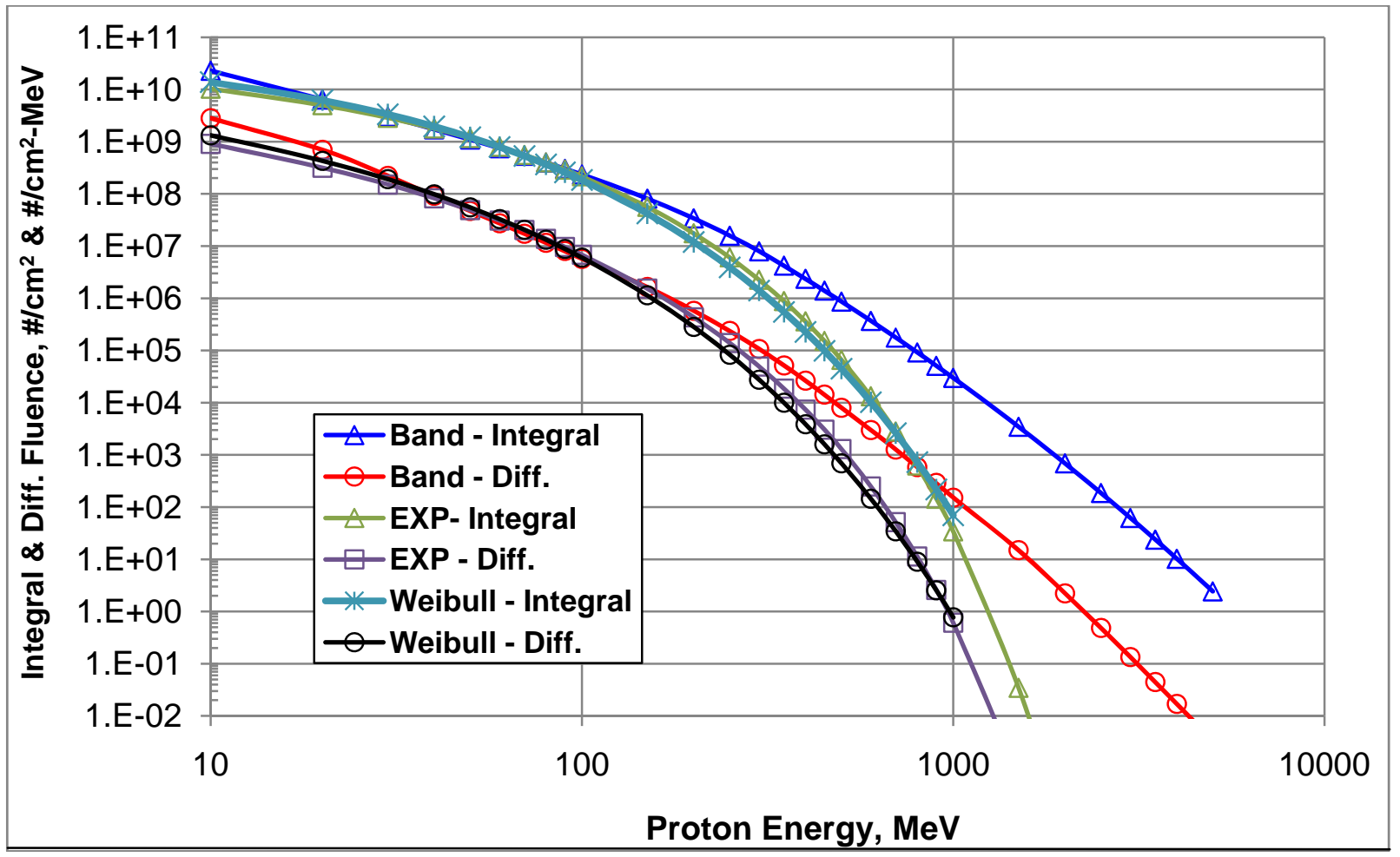

Figure 1 (l) 2001 Nov 4 


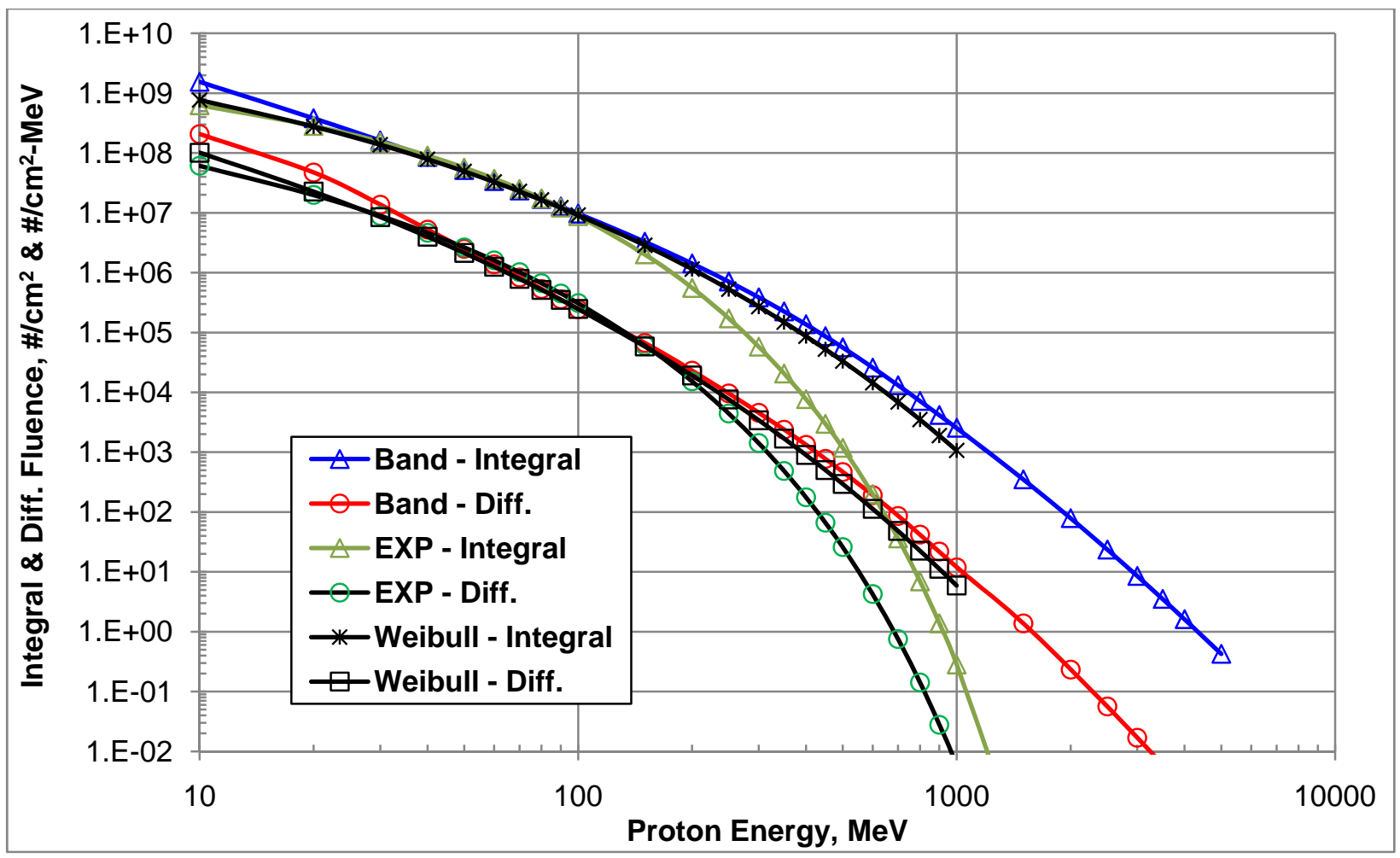

Figure 1 (m) 2003 Nov 2

\section{Radiation Exposure Calculations}

We now present radiation exposure calculations for the 12 GLEs plus the exposures for the combined $12 \& 15$ November 1960 events. Using the NASA LaRC HZETRN $2010^{6}$ code and the differential spectra for the dozen plus

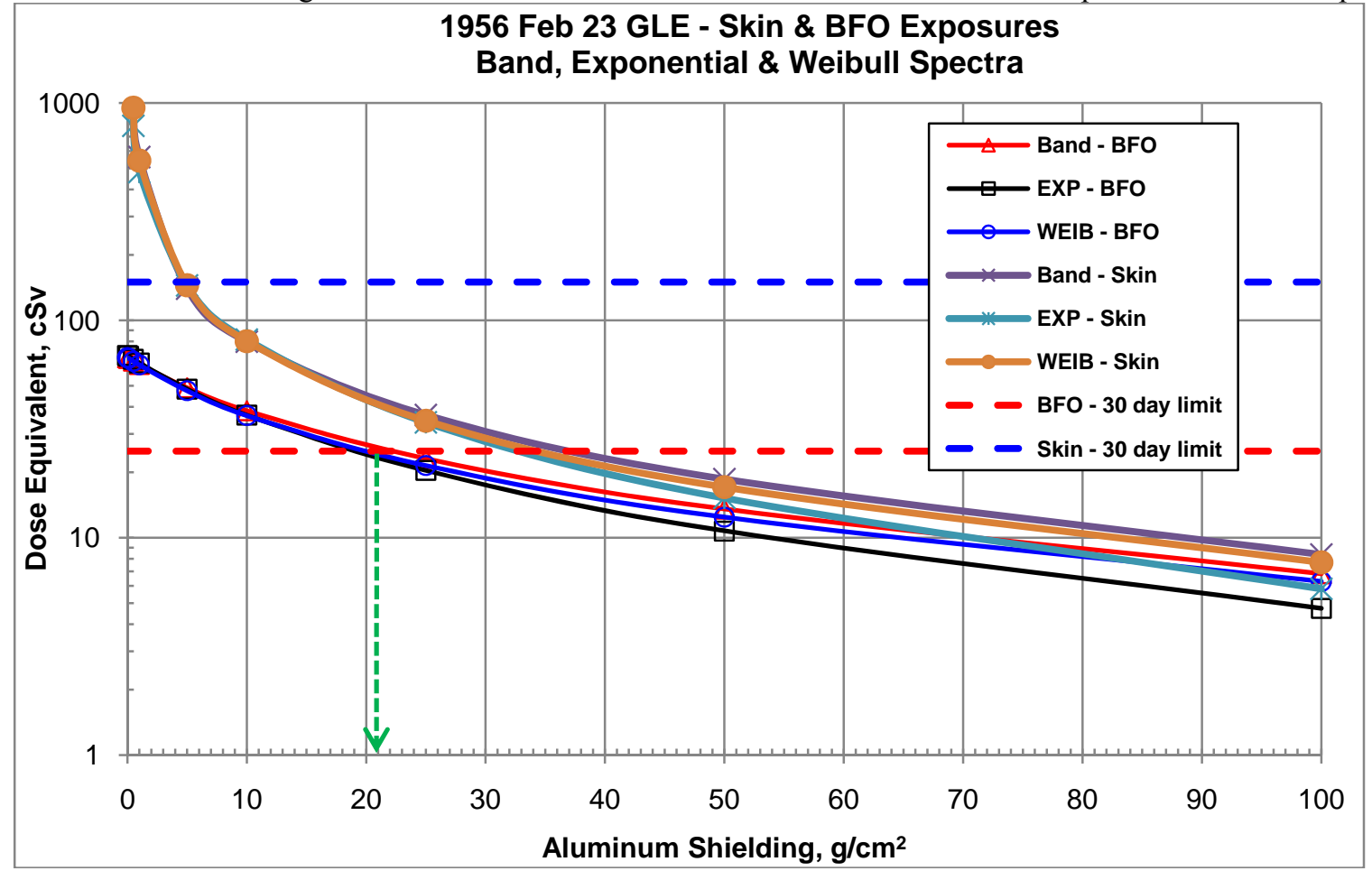

Figure 2 (a) 1956 Feb 23 GLE 
GLEs, the skin and BFO exposures were computed and compared with the 30-day and annual NASA crew exposure limits. These are shown in Figures 2. (a) - (m). The green dash line indicates the amount of aluminum shielding required to not exceed the BFO 30-day limit for some of the events.

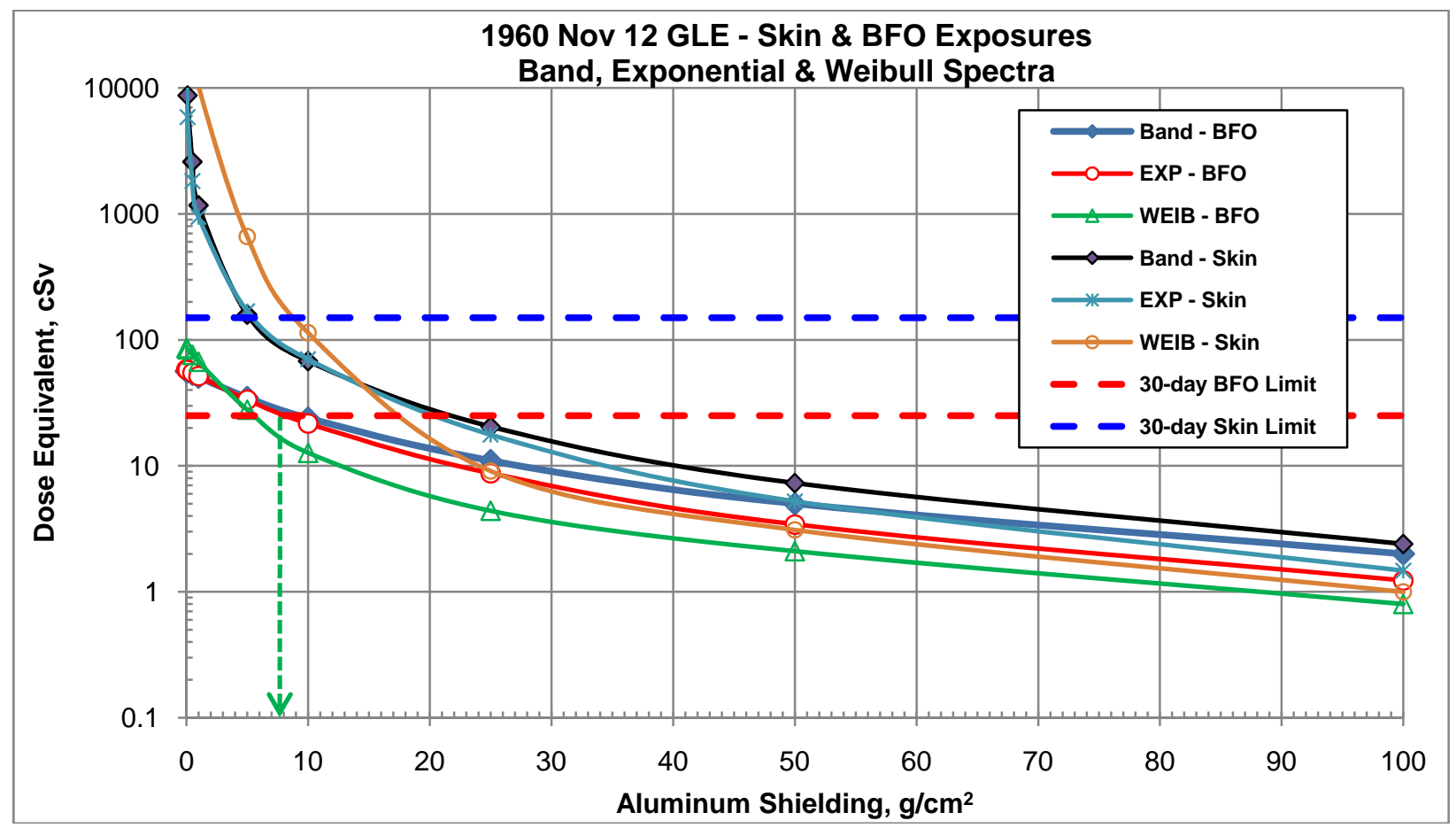

Figure 2 (b) 1960 Nov 12 GLE

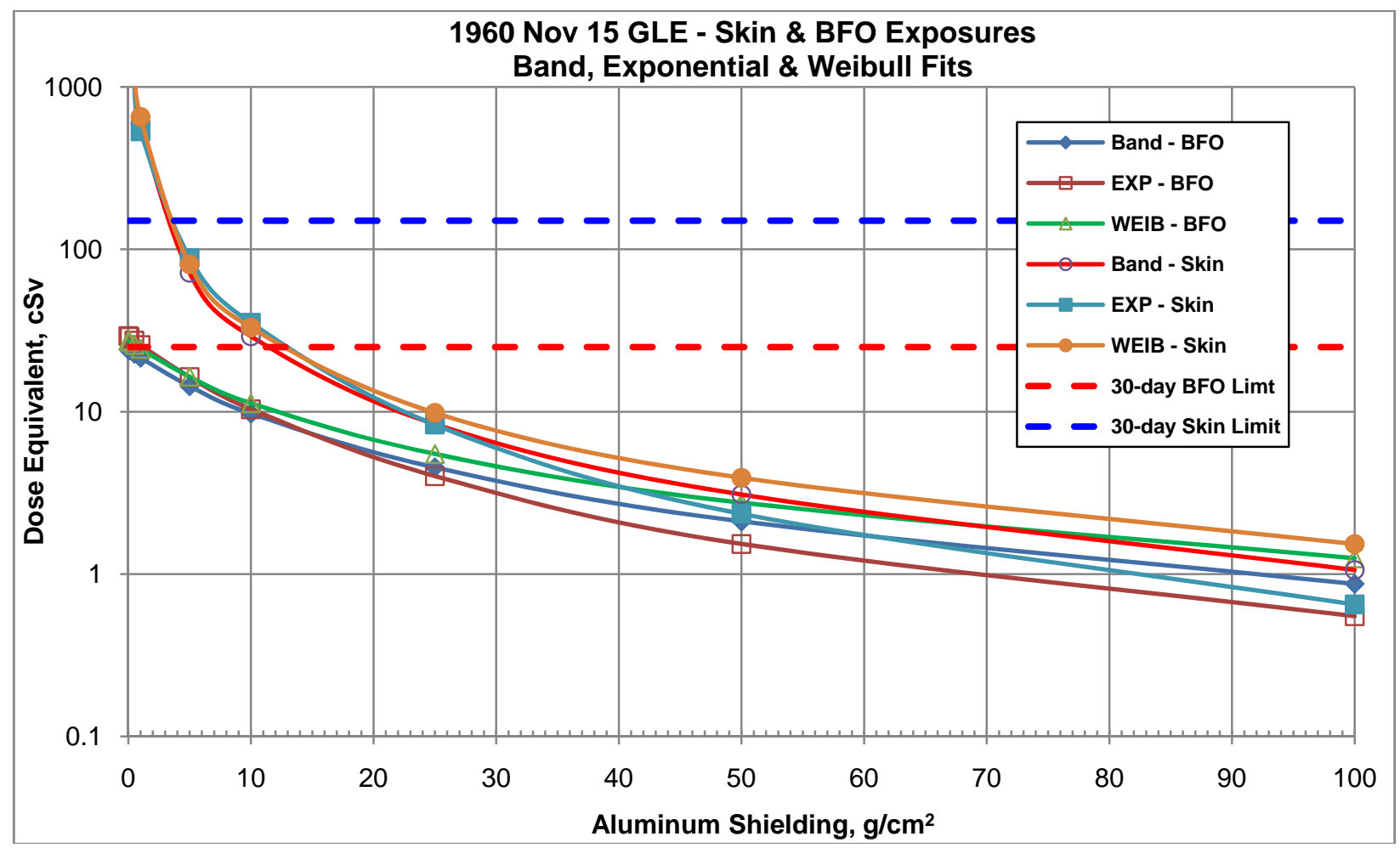

Figure 2 (c) 1960 Nov 15 GLE 


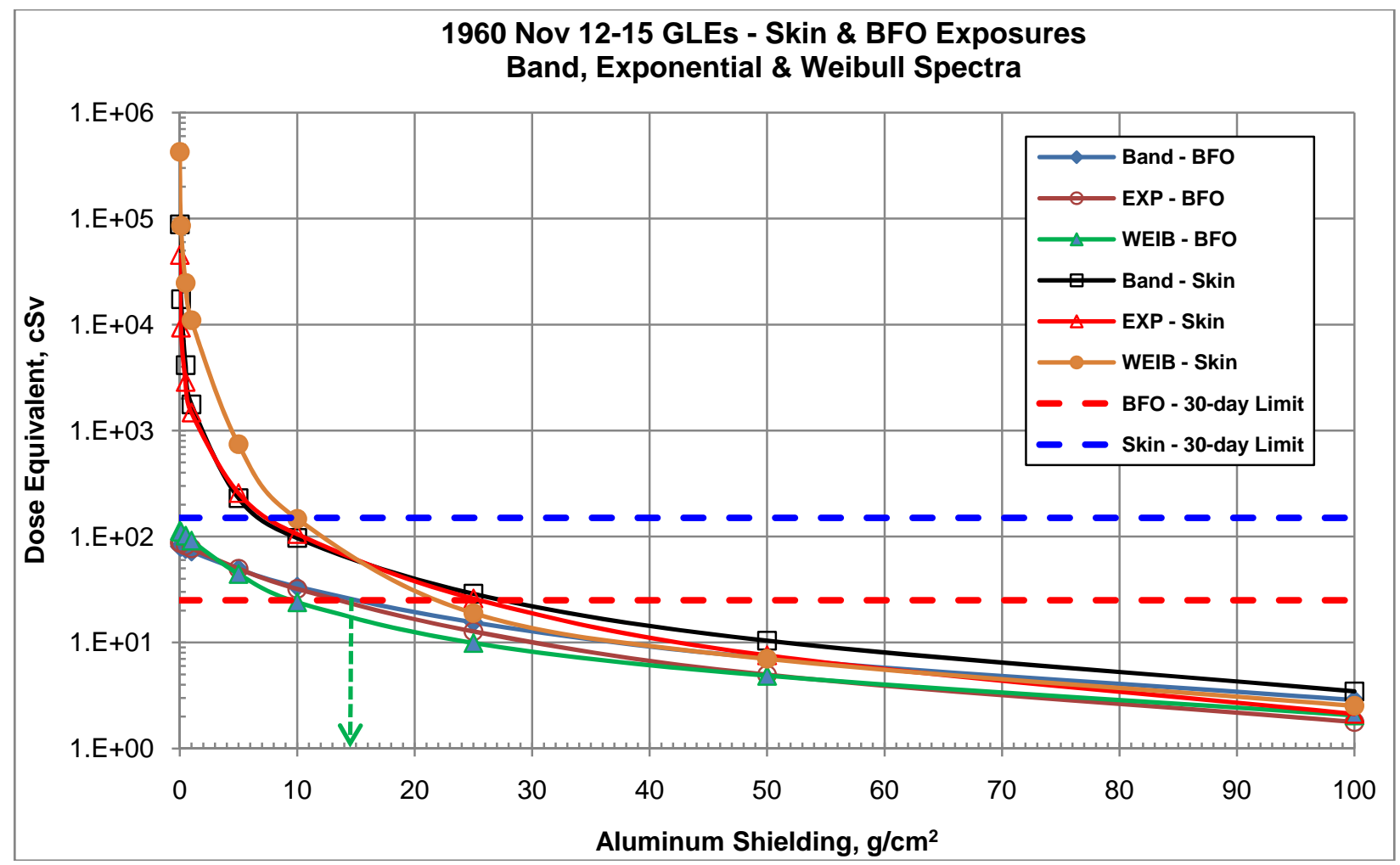

Figure 2 (d) 1960 Nov 12-15

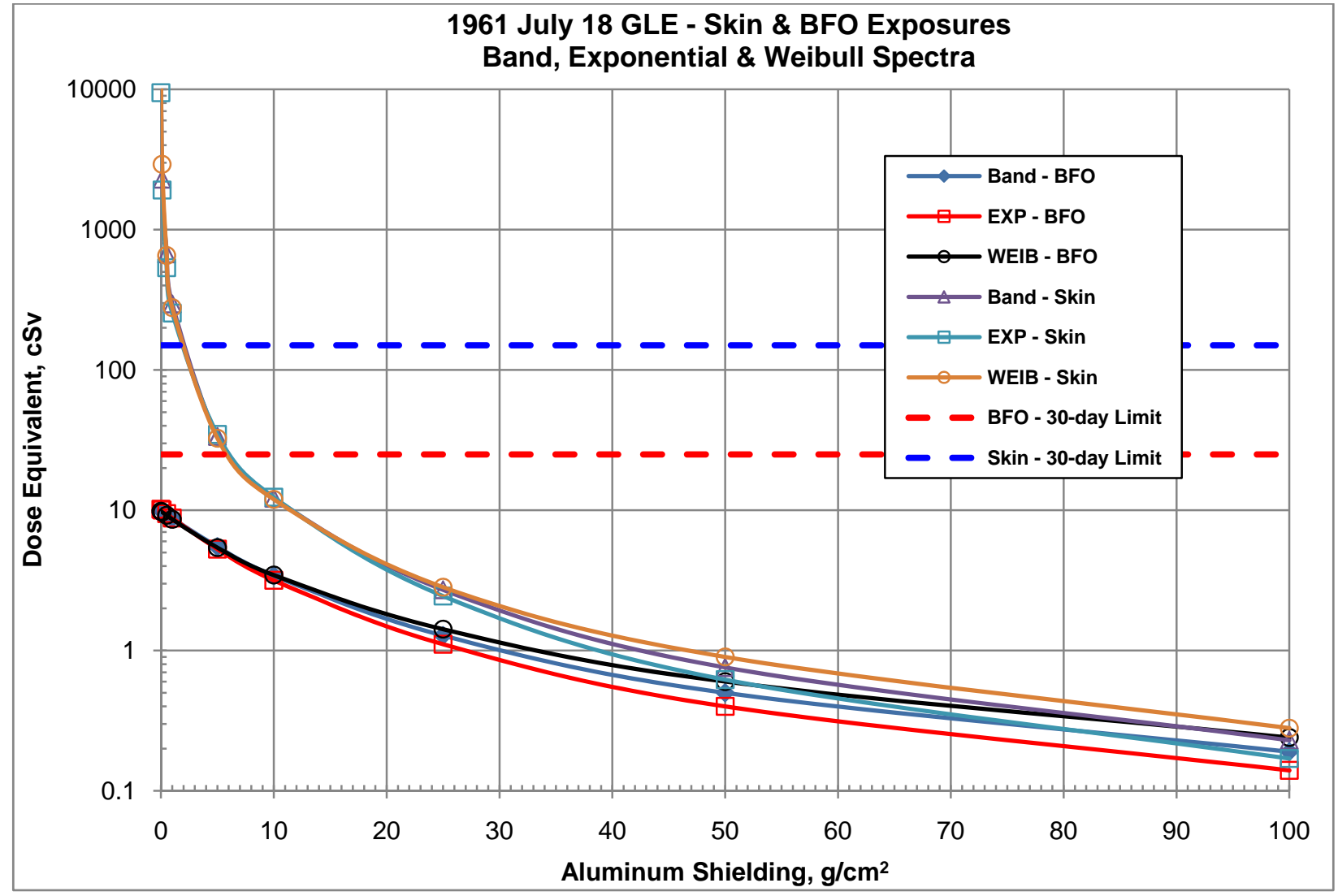

Figure 2 (e) 1961 July 18 


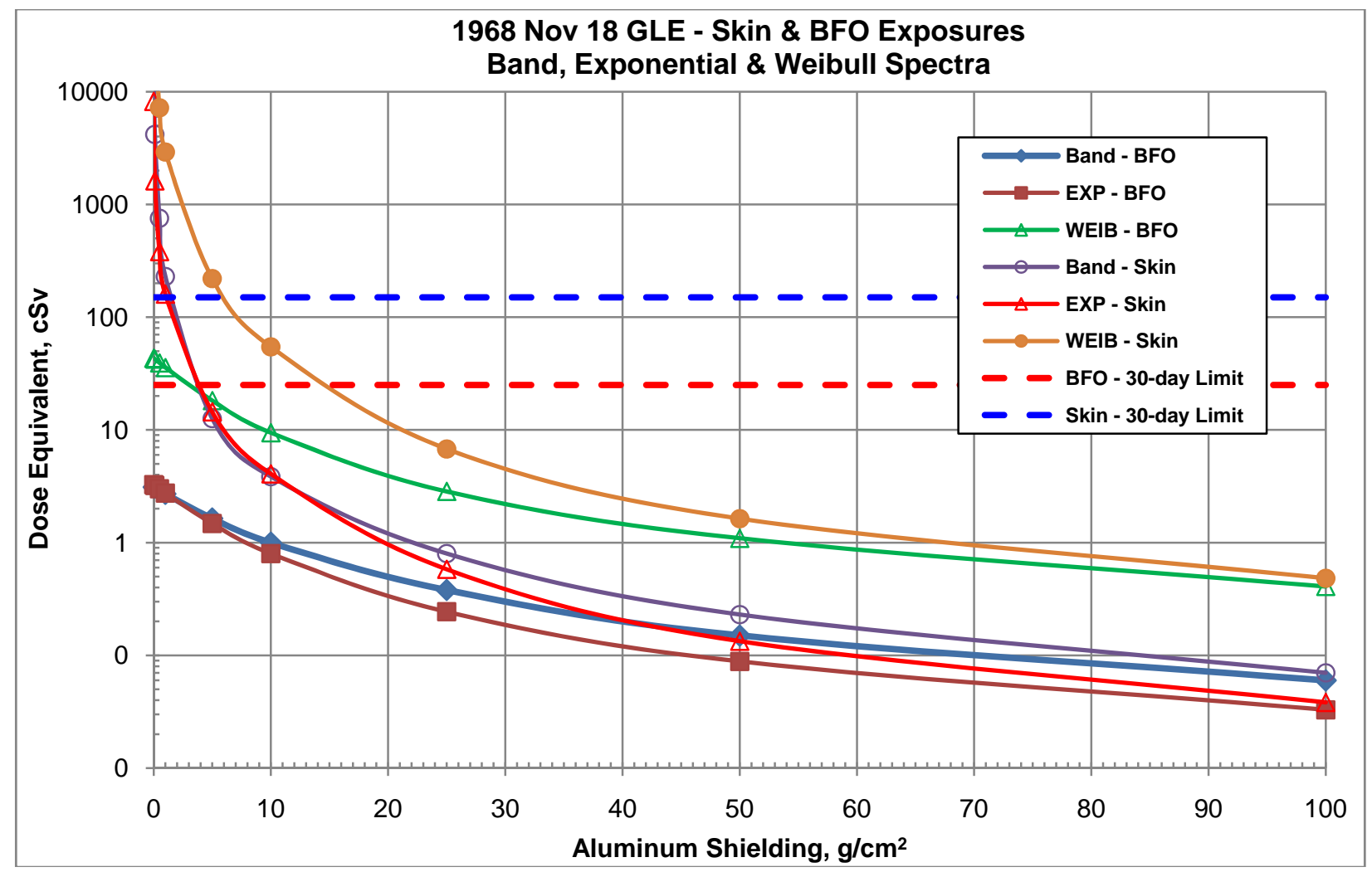

Figure 2 (f) 1968 Nov 18

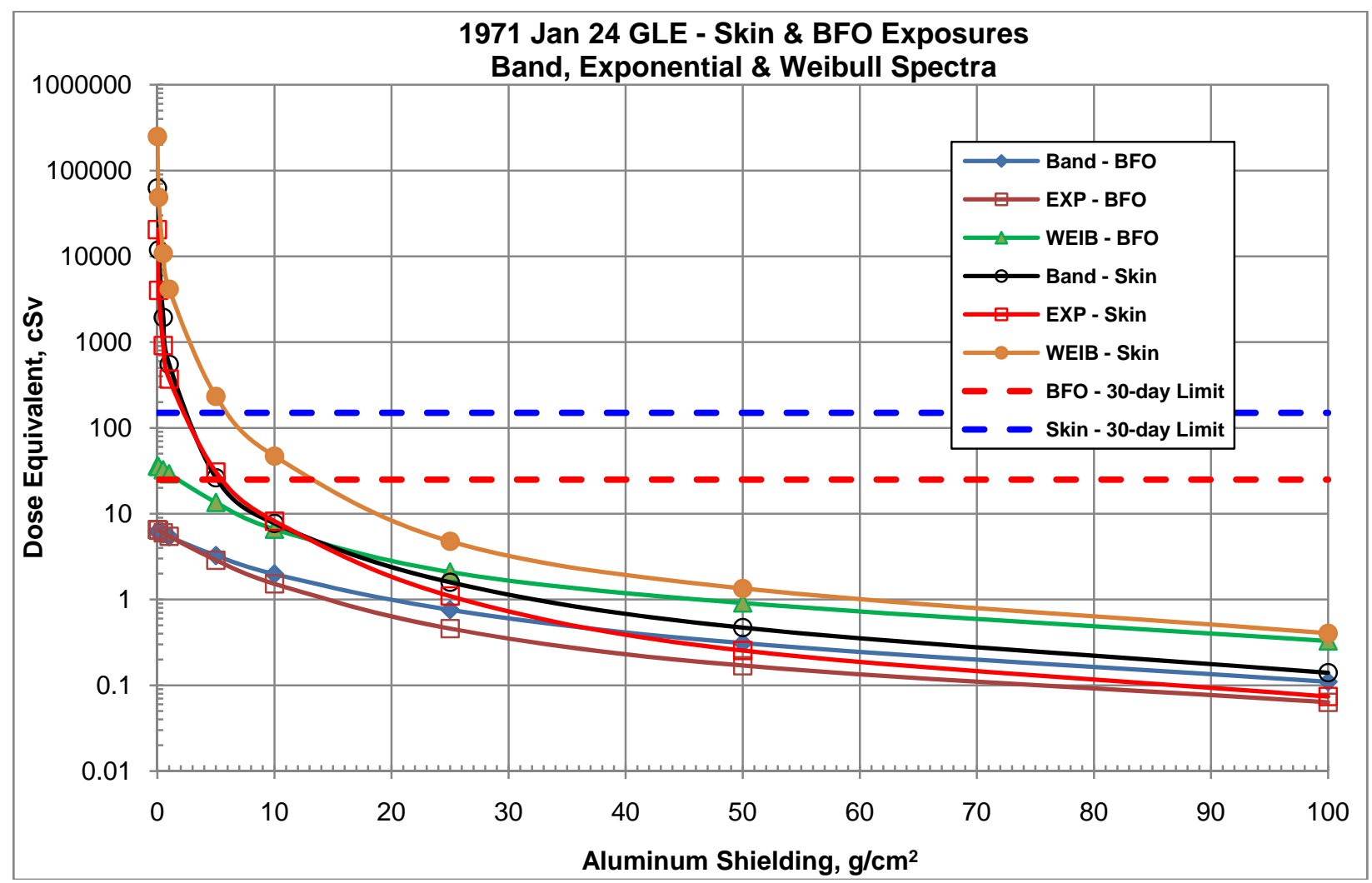

Figure 2 (g) 1971 Jan 24 


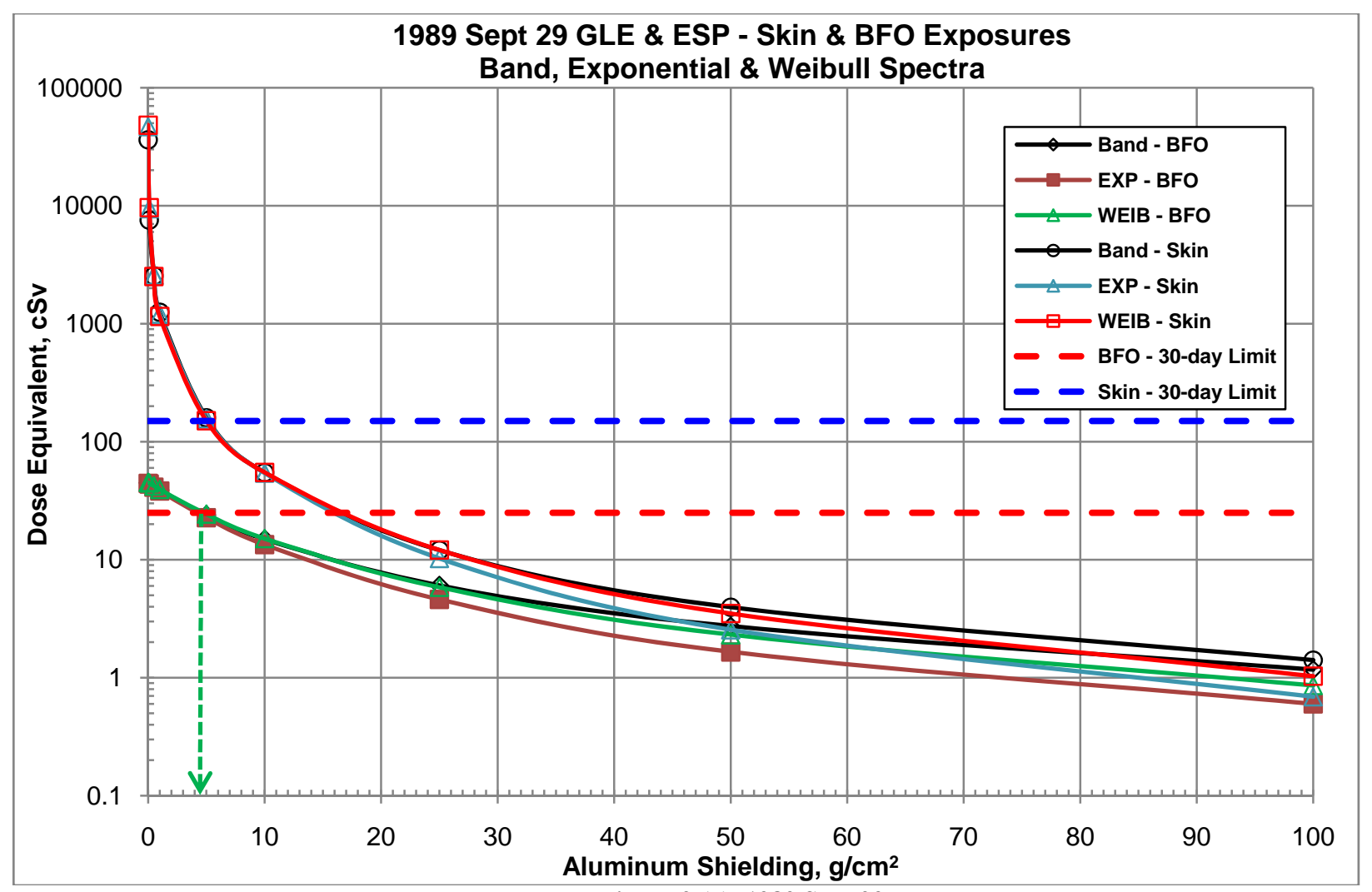

Figure 2 (h) 1989 Sept 29

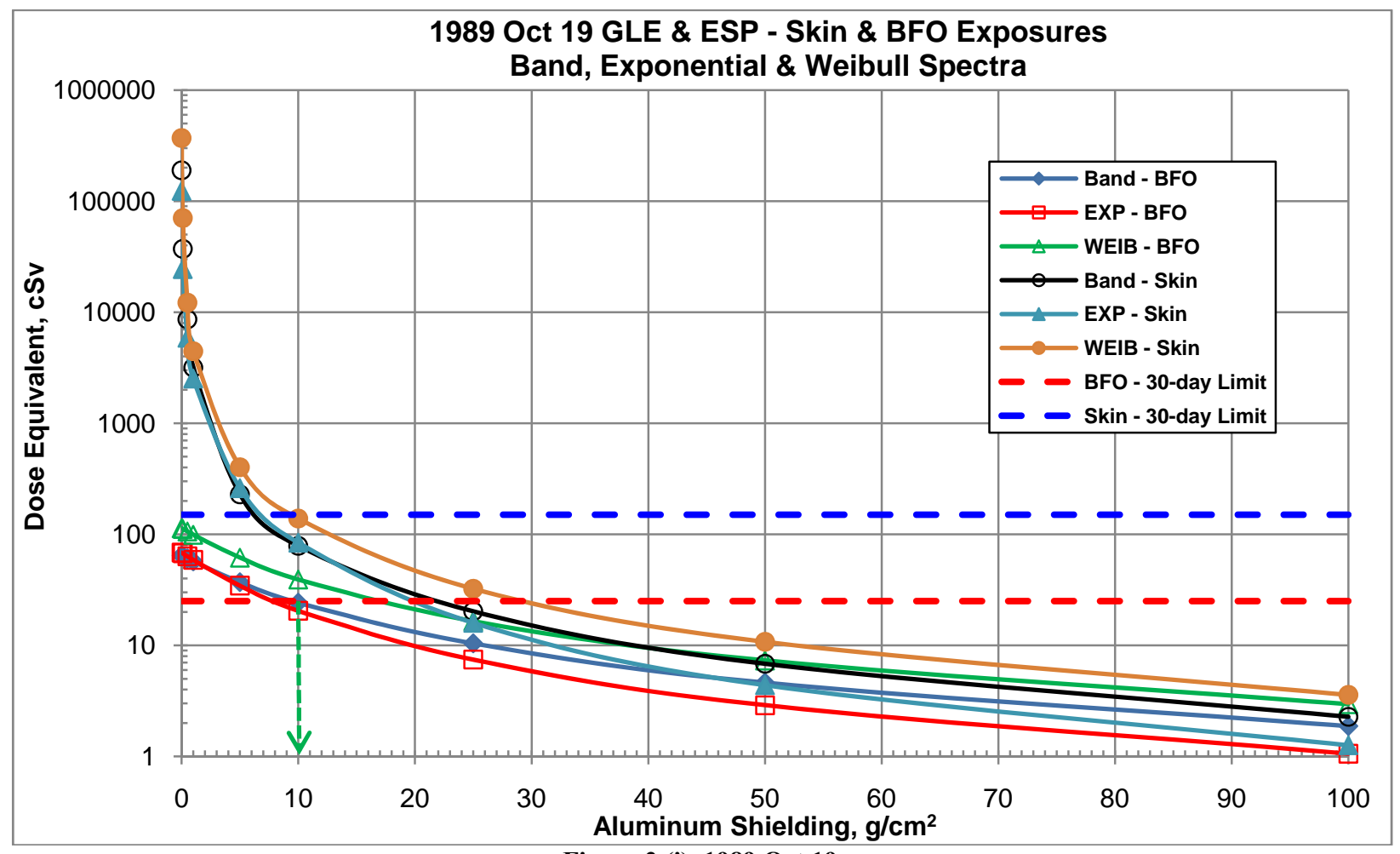

Figure 2 (i) 1989 Oct 19 


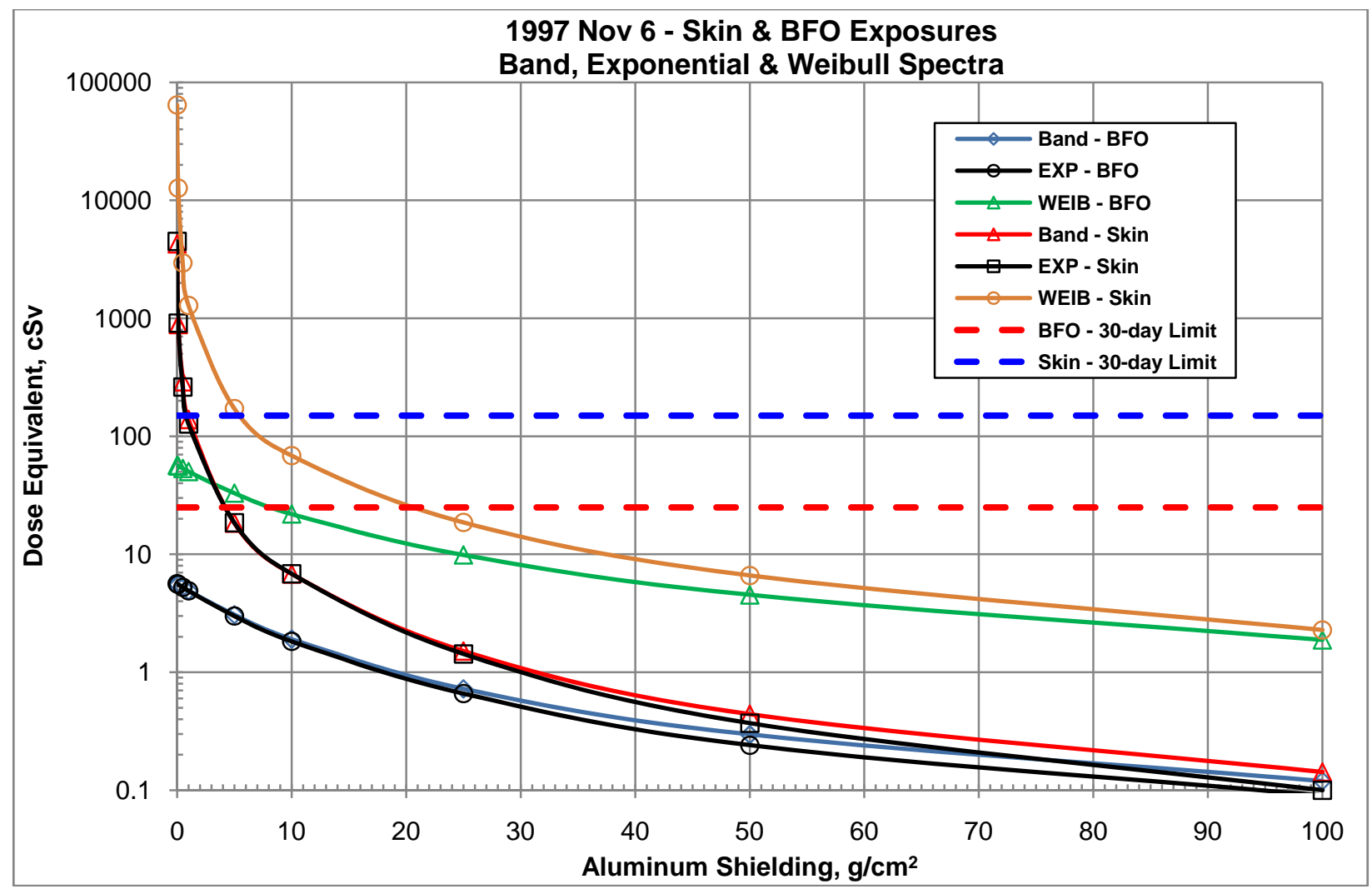

Figure 2 (j) 1997 Nov 6

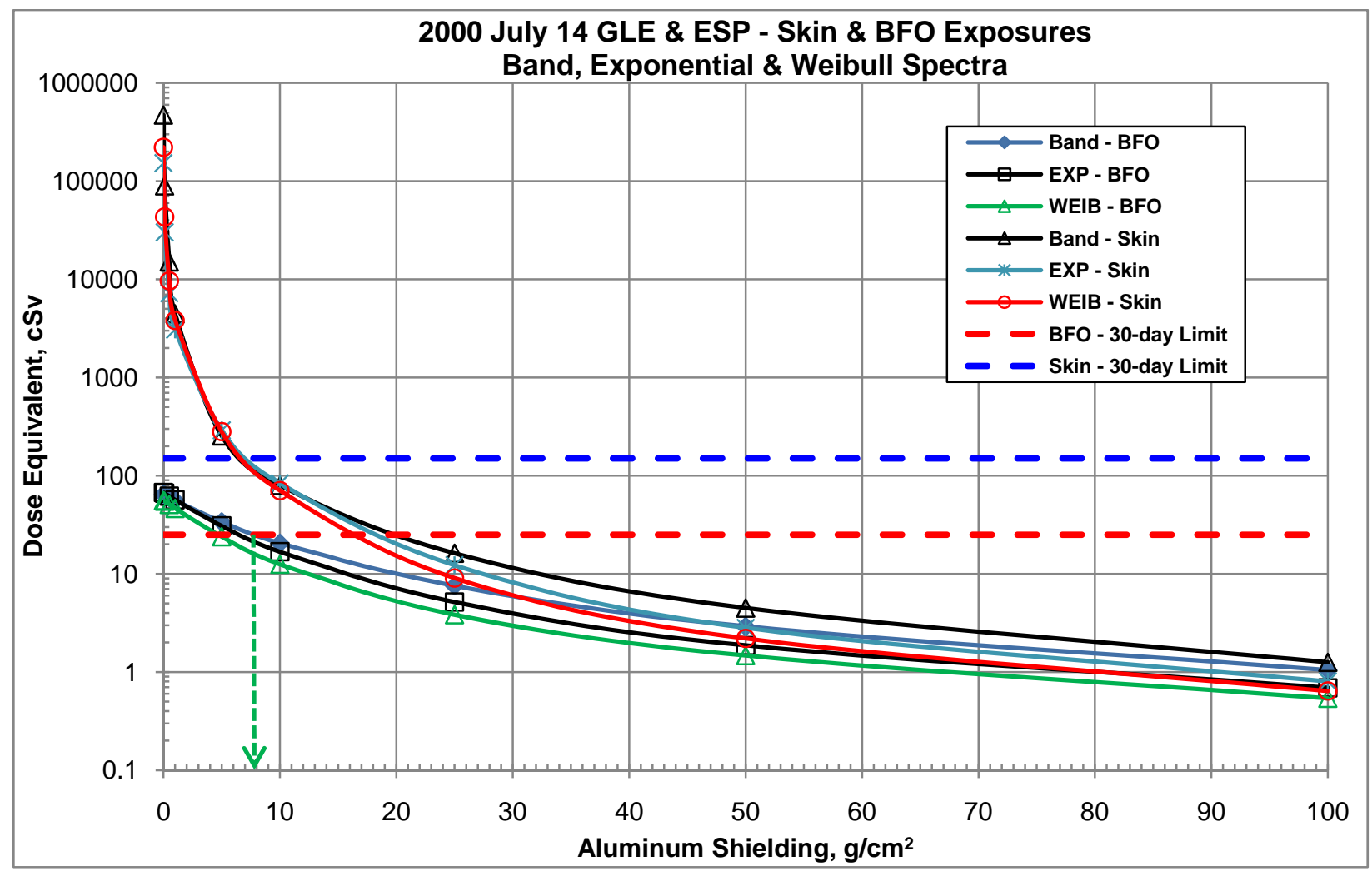

Figure 2 (k) 2000 July 14 


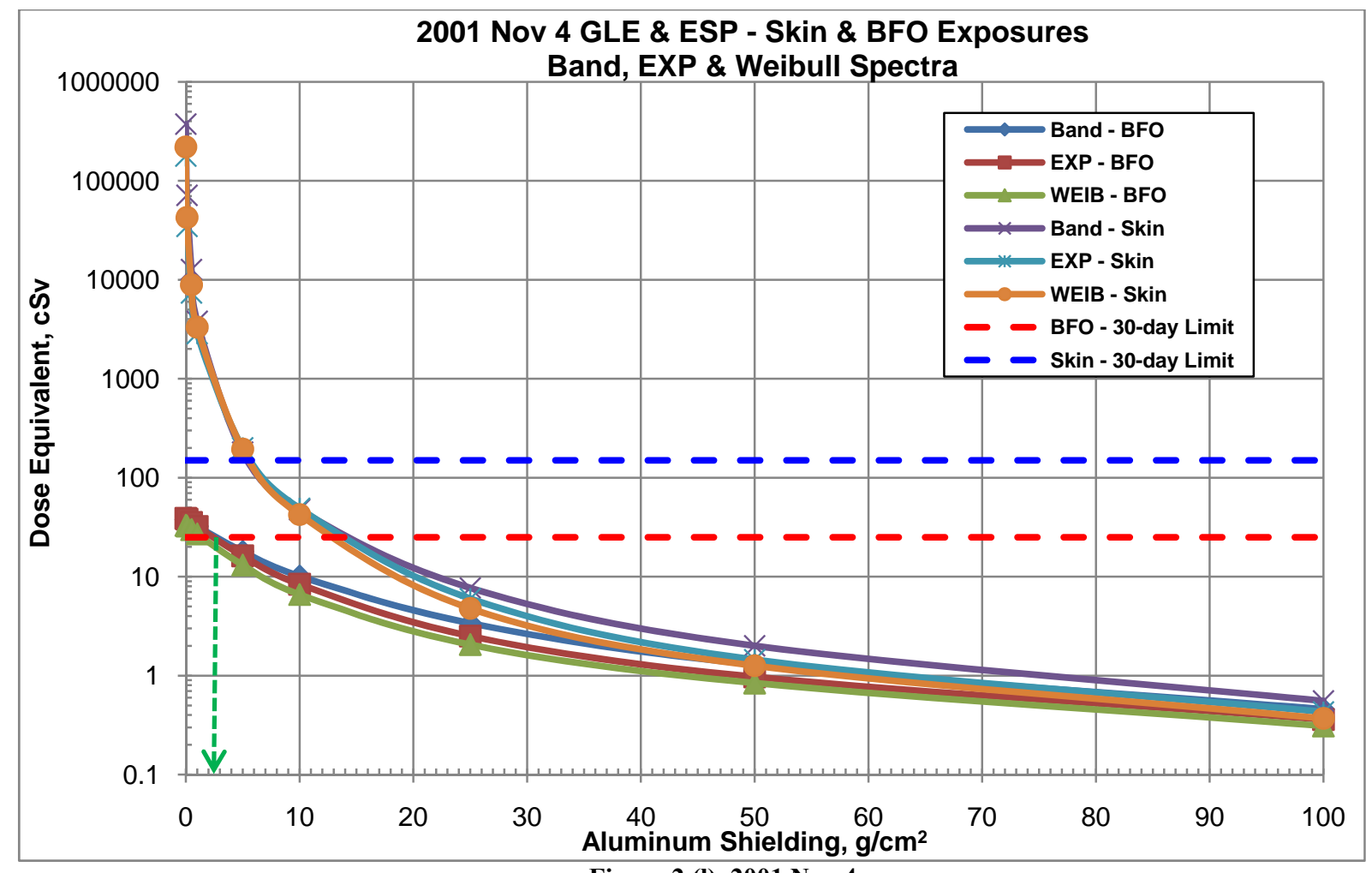

Figure 2 (I) 2001 Nov 4

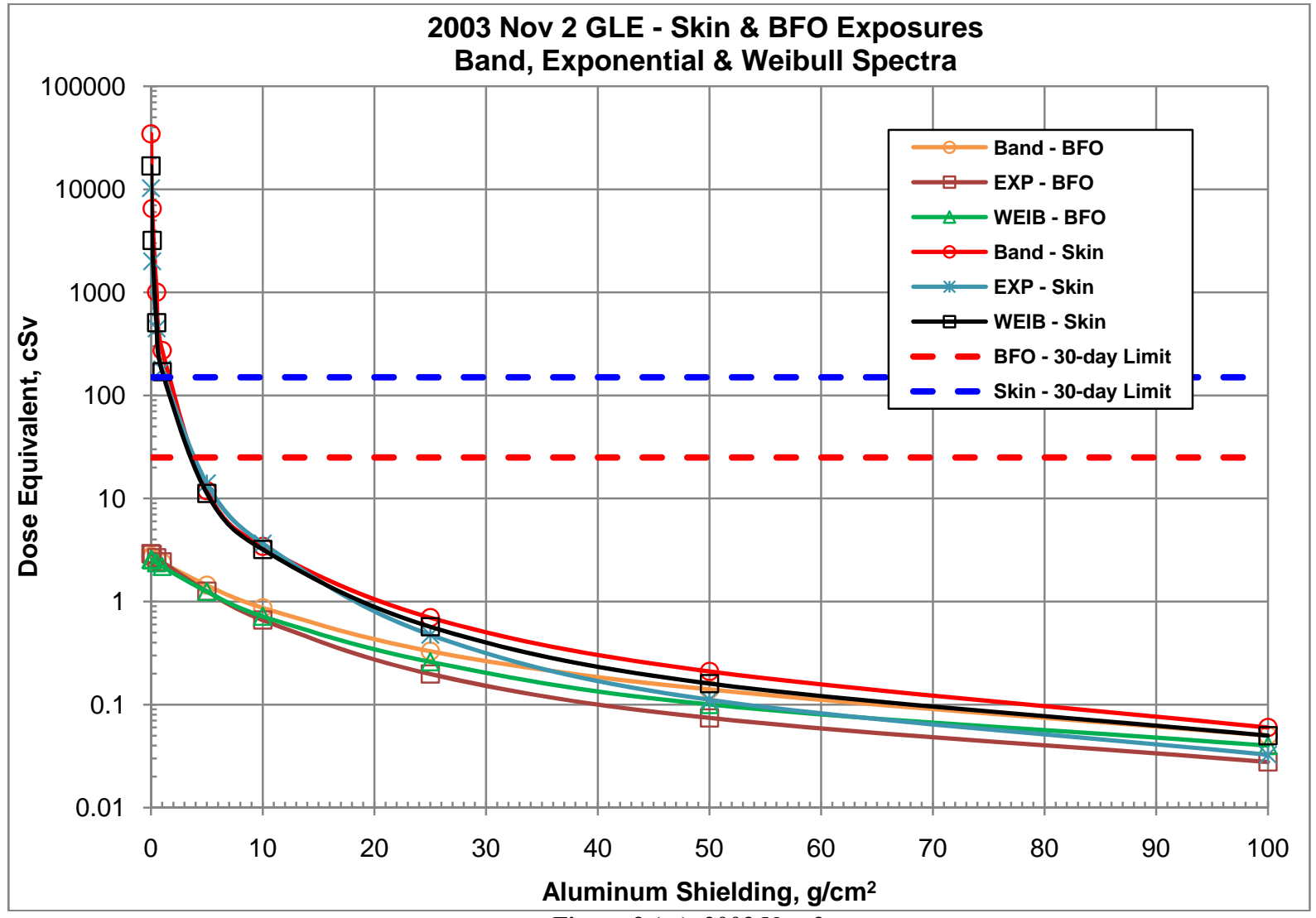

Figure 2 (m) 2003 Nov 2 


\section{Conclusions}

A number of conclusions can be made from the previous assessments for the 12 GLEs selected for this study:

1) The 12 GLEs selected for this study were made since it provided a means of comparison with the Band and Weibull fitting methods.

2) Three spectral fitting methods, Band, exponential and Weibull, were compared; in all 12 cases the exponential in proton rigidity fitting method grossly under-estimated the other two methods and consequently grossly under-estimated the dose equivalent.

3) The Band method completely describes the proton spectrum over the entire energy range from $10 \mathrm{MeV}$ to $5 \mathrm{GeV}$.

4) Only one case, 1956 February 23, showed a reasonable comparison between the Band and Weibull spectral fitting methods; for the other 11 cases the Weibull method varied considerably when compared with the Band method.

5) Skin and BFO dose equivalent values for the 12 GLEs were computed using the NASA Langley Research Center high energy particle transport/dose code; the values were compared with the NASA crew exposure limits for 30-days and an annual limit.

6) Of the 12 GLEs analyzed, four GLEs (1960 November 12, 1968 November 18, 1971 January 24, and 1997 November 6) produced an order of magnitude or higher proton fluences, when using the Weibull method:

a. For the 1960 November 12 event, the Weibull method produced $\sim$ a factor of 10 higher proton fluence for energies $<100 \mathrm{MeV}$ when compared with the Band method.

b. For the 1968 November 18 event, the Weibull method produced $\sim$ a factor of 10 higher proton fluence for energies $<400 \mathrm{MeV}$ when compared with the Band method.

c. For the 1971 January 24 event, the Weibull method produced $\sim$ a factor of 10 higher proton fluence for energies $<200 \mathrm{MeV}$ when compared with the Band method.

d. For the 1997 November 6 event, the Weibull method produced $\sim$ a factor of 10 higher proton fluence for energies $<1000 \mathrm{MeV}(1 \mathrm{GeV})$ when compared with the Band method.

7) Seven of the 12 GLEs required additional aluminum shielding ranging from $2.5-22.0 \mathrm{~g} / \mathrm{cm}^{2}$ aluminum to stay at or below the 30-day BFO crew exposure limit as shown in Figs. 2 (a), 2 (b), 2 (d), 2 (h), 2 (i), $2(\mathrm{k})$, and $2(\mathrm{l})$.

8) We strongly recommend that the Band function spectral fitting method be utilized by the scientific community for analyzing any future GLEs.

\section{References}

${ }^{\mathbf{1}}$ Malitson, H. H., and W. R. Webber, Characteristics of solar cosmic ray events and correlation with solar flare data, NASA Goddard Space Flight Center, Greenbelt, MD, 1963.

${ }^{2}$ Xapsos, et al., Characterizing solar proton energy spectra for radiation effects applications. IEEE Trans. Nucl. Sci. 47, 22182223, Dec. 2000.

${ }^{3}$ Allan Tylka and William Dietrich, "Proton Spectra in Ground-Level Enhanced (GLE) Solar Particle Events," $37^{\text {th }}$ Committee on Space Research (COSPAR) Scientific Assembly, Session D23-0003-08, Montreal, Canada, 2008.

${ }^{4}$ William Atwell, et al., "Radiation Exposure Estimates for Extremely Large Solar Proton Events," $37^{\text {th }}$ Committee on Space Research (COSPAR) Scientific Assembly, Montreal, Canada, Session F25-0027-08, 2008.

${ }^{\mathbf{5}}$ Band, D., et al., "BATSE Observations of gamma-ray burst spectra I. - Spectral diversity", Astrophysical Journal, 413, 281 292, 1993.

${ }^{6}$ HZETRN 2010, computer code with limited distribution by NASA LaRC, Hampton, VA, 2010.

${ }^{7}$ SigmaStat version 2.03. Chicago, IL: SPSS Inc., 1995.

${ }^{8}$ W. H. Press, S. A. Teukolsky, W. T. Vettering, and B. P. Flannery, Numerical Recipes in Fortran-The Art of Scientific Computing, 2nd ed. New York, NY: Cambridge University Press, 1992.

${ }^{9}$ Myung-Hee Y. Kim, Matthew J. Hayat, Alan H. Feiveson, and Francis A. Cucinotta, Prediction of frequency and exposure level of solar particle events, Health Physics Journal, Volume 97 - Issue 1 - pp 68-81, July 2009.

${ }^{10}$ Kim, Myung-Hee, William Atwell, Allan J. Tylka, William F. Dietrich, and Francis A. Cucinotta, "Radiation Dose Assessments of Solar Particle Events with Spectral Representation at High Energies for the Improvement of Radiation Protection," $38^{\text {th }}$ Committee on Space Research (COSPAR) Scientific Assembly, Session F2.4, Bremen, Germany, 2010. 